Geometry $\&$ Topology

Volume 8 (2004) 1043-1078

Published: 18 August 2004

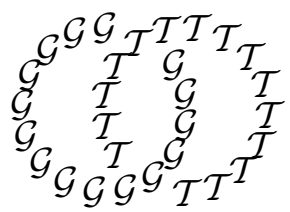

\title{
The surgery obstruction groups of the infinite dihedral group
}

\author{
Francis X Connolly \\ JAMES F DAVIS \\ Department of Mathematics, University of Notre Dame \\ Notre Dame, IN 46556, USA \\ and \\ Department of Mathematics, Indiana University \\ Bloomington, IN 47405, USA
}

Email: connolly.1@nd.edu and jfdavis@indiana.edu

\begin{abstract}
This paper computes the quadratic Witt groups (the Wall $L$-groups) of the polynomial ring $\mathbb{Z}[t]$ and the integral group ring of the infinite dihedral group, with various involutions. We show that some of these groups are infinite direct sums of cyclic groups of order 2 and 4 . The techniques used are quadratic linking forms over $\mathbb{Z}[t]$ and Arf invariants.
\end{abstract}

\section{AMS Classification numbers Primary: 57R67}

Secondary: 19J25, 19G24

Keywords: Surgery, infinite dihedral group, Gauss sums

Proposed: Steven Ferry

Seconded: Benson Farb, Ralph Cohen
Received: 5 June 2003

Accepted: 11 July 2004 


\section{Introduction and statement of results}

In this paper we complete the computation of the Wall surgery obstruction groups for the infinite dihedral group, the $L$-theory of the polynomial ring $\mathbb{Z}[t]$, the $L$-theory of the Laurent polynomial ring $L_{n}\left(\mathbb{Z}\left[t, t^{-1}\right]\right)$, with either the trivial involution or the involution $t \mapsto-t$, and the Cappell unitary nilpotent groups for the ring $\mathbb{Z}$. The problem of computing these groups is thirty years old. We take an historical approach in this introduction which sets the stage and indicates the interrelation between the various groups, but has the drawback of postponing the discussion of the main results of this paper. The main results are 1.10, 1.9, 1.7 and 1.8.

Our algebraic computations are motivated by the following geometric question: is a homotopy equivalence

$$
f: M \rightarrow X_{1} \# X_{2}
$$

from a closed $n$-manifold to a connected sum of two others splittable? That is to say, is $M$ expressible as a connected sum $M=M_{1} \# M_{2}$ so that $f$ homotopic to a map of the form

$$
f_{1} \# f_{2}: M_{1} \# M_{2} \rightarrow X_{1} \# X_{2}
$$

where each $f_{i}: M_{i} \rightarrow X_{i}$ is a homotopy equivalence? In particular, is $M$ itself a connected sum?

Let's restrict now to the case where both $X_{i}$ are connected and have cyclic fundamental group of order two. Cappell [5, 8] defined an element $s(f)$ in a $4-$ periodic abelian group $\mathrm{UNil}_{n+1}\left(\mathbb{Z} ; \mathbb{Z}^{ \pm}, \mathbb{Z}^{ \pm}\right)$. The \pm 's depend on the orientability of $X_{1}$ and $X_{2}$ and are often omitted if both are orientable. If $s(f) \neq 0$, then $f$ is not splittable. If $s(f)=0$ and $n \geq 4$, then $f$ is splittable topologically; if $s(f)=0$ and $n \geq 5$, then $f$ splits smoothly. (In the smooth case one allows connected sum along a homotopy sphere.) Fixing $X_{1}$ and $X_{2}$ and given $s \in \mathrm{UNil}_{n+1}\left(\mathbb{Z} ; \mathbb{Z}^{ \pm}, \mathbb{Z}^{ \pm}\right)$, there is a realization result: there is a homotopy equivalence $f: M \rightarrow X_{1} \# X_{2}$ with $s(f)=s$, with $M$ a topological manifold when $n \geq 4$, a smooth manifold when $n \geq 5$. A particularly interesting example is to take $X_{1}=X_{2}=\mathbb{R} P^{4}$ and $0 \neq s \in \operatorname{UNil}_{5}\left(\mathbb{Z} ; \mathbb{Z}^{-}, \mathbb{Z}^{-}\right)$; then realization gives a nonsplittable homotopy equivalence $M \rightarrow \mathbb{R} P^{4} \# \mathbb{R} P^{4}$. The geometric properties of $M$ seem both unexplored and of some interest.

The unitary nilpotent groups $\operatorname{UNil}_{n}\left(R ; A_{1}, A_{2}\right)$ are defined for a ring $R$ with involution and $R$-bimodules $A_{1}, A_{2}$ with involution. We won't need the definitions of these groups, only their relation to $L$-groups discussed below and the 
isomorphisms

$$
\mathrm{UNil}_{n}\left(R ; A_{2}, A_{1}\right) \cong \operatorname{UNil}_{n}\left(R ; A_{1}, A_{2}\right) \cong \operatorname{UNil}_{n+2}\left(R ; A_{1}^{-}, A_{2}^{-}\right) .
$$

Here $A_{i}^{-}$is the bimodule $A_{i}$, but with the involution $a \mapsto-\bar{a}$.

Associated to a ring with involution are the algebraic $L$-groups $L_{n}(R)$. They are 4-periodic. The definition of $L_{0}(R)$ (and $L_{2}(R)$ ) are reviewed in Section 2, they are Witt groups of (skew)-quadratic forms. $L_{2 k+1}(R)$ is the abelianization of the stable automorphism group of the $(-1)^{k}$-hyperbolic form (any form admitting a Lagrangian) 1

Fix a group $G$ and a homomorphism $w: G \rightarrow\{ \pm 1\}$. There is an induced involution $\sum a_{g} g \mapsto \sum a_{g} w(g) g^{-1}$ on $\mathbb{Z} G$. The associated groups $L_{n}(\mathbb{Z} G, w)$ are key ingredients in the classification of closed, oriented manifolds with fundamental group $G$ and orientation character $w$.

Parallel to the work of Stallings [26] and Waldhausen [27] in algebraic $K-$ theory, Cappell [7, 9] studied the $L$-groups of amalgamated free products and showed that if $H$ is a subgroup of groups $G_{1}$ and $G_{2}$, then $\operatorname{UNil}_{n}\left(\mathbb{Z} H ; \mathbb{Z}\left[G_{1}-\right.\right.$ $\left.H], \mathbb{Z}\left[G_{2}-H\right]\right)$ is a summand of $L_{n}\left(\mathbb{Z}\left[G_{1}{ }_{H} G_{2}\right]\right)$, and that the $L$-group modulo the UNil-term fits into a Mayer-Vietoris exact sequence. Farrell [12] showed that the UNil-term has exponent at most four. However he was unable to find an element $\alpha \in \operatorname{UNil}_{n}(R ; A, B)$ for which $2 \alpha \neq 0$. Cappell proved that the UNil-term vanishes provided that the inclusions $H \hookrightarrow G_{i}$ are square root closed, ie, $g \in G_{i}$ and $g^{2} \in H$ implies $g \in H$.

The infinite dihedral group is

$$
D_{\infty}=\mathbb{Z}_{2} * \mathbb{Z}_{2}=\left\langle a_{1}, a_{2} \mid a_{1}^{2}=e=a_{2}^{2}\right\rangle=\left\langle g, t \mid t^{2}=e, t g t^{-1}=g^{-1}\right\rangle .
$$

Let $w: D_{\infty} \rightarrow\{ \pm 1\}$ be a homomorphism. The $L$-groups $L_{n}\left(\mathbb{Z}\left[D_{\infty}\right], w\right)$ and the corresponding $\mathrm{UNil-groups} \mathrm{UNil}_{n}\left(\mathbb{Z} ; \mathbb{Z}^{w\left(a_{1}\right)}, \mathbb{Z}^{w\left(a_{2}\right)}\right)$ seem particularly fundamental. First the infinite dihedral group is the simplest group which is not square root closed. Second, due to the isomorphism conjecture of Farrell--Jones 13. (generalizing the Borel-Novikov conjectures of manifold theory), attention has been recently focused on the infinite dihedral group. The isomorphism conjecture roughly states that $L_{n}(\mathbb{Z} \Gamma)$ depends on the $K$ - and $L$-theory of virtually cyclic subgroups and homological data depending on $\Gamma$. A group $G$ is

\footnotetext{
${ }^{1}$ These are the so-called $L^{h}$-groups measuring the obstruction to doing surgery up to homotopy equivalence. They are defined as in Wall [29] Chapters 6 and 7], except one deletes the requirement that the torsions are trivial. A definition of these groups is given in [16] where they were denoted $V_{n}(R)$. Ranicki 20] later gave a definition of $L_{n}(R)$ as cobordism classes of $n$-dimensional quadratic Poincaré complexes over $R$.
} 
virtually cyclic if either $G$ is finite, or $G$ surjects onto $\mathbb{Z}$ with finite kernel, or $G$ surjects onto $D_{\infty}$ with finite kernel. The $L$-theory in the first two cases has been examined in detail [14, 25, 17, 18]. Therefore $L_{n}\left(D_{\infty}\right)$ is the next obvious case to consider.

In this paper we are writing a conclusion to the long tale of the computation of $\mathrm{UNil}_{n}\left(\mathbb{Z} ; \mathbb{Z}^{ \pm}, \mathbb{Z}^{ \pm}\right)$. Cappell showed $\mathrm{UNil}_{2}(\mathbb{Z} ; \mathbb{Z}, \mathbb{Z})$ was infinitely generated [6] and announced that $\operatorname{UNil}_{0}(\mathbb{Z} ; \mathbb{Z}, \mathbb{Z})=0$ [7]. Connolly-Koźniewski 10] obtained an isomorphism $\mathrm{UNil}_{2}(\mathbb{Z} ; \mathbb{Z}, \mathbb{Z}) \cong\left(\mathbb{Z}_{2}\right)^{\infty}$ and also showed that $\mathrm{UNil}_{0}(\mathbb{Z} ; \mathbb{Z}, \mathbb{Z})=0$. Connolly-Ranicki 11 showed $\operatorname{UNil}_{1}(\mathbb{Z} ; \mathbb{Z}, \mathbb{Z})=0$ and computed $\mathrm{UNil}_{3}(\mathbb{Z} ; \mathbb{Z}, \mathbb{Z})$ up to extension, and thereby showed that it was infinitely generated. Andrew Ranicki outlined the construction and detection of an element of $\mathrm{UNil}_{3}(\mathbb{Z} ; \mathbb{Z}, \mathbb{Z})$ of exponent 4 in a letter $[22$ to the first author. After a preliminary version of this paper was circulated, Banagl-Ranicki 3] gave an independent complete computation of $\operatorname{UNil}_{3}(\mathbb{Z} ; \mathbb{Z}, \mathbb{Z})$ using generalized Arf invariants.

In this paper we give complete computations for all $n$ as well as doing the non-oriented case.

But before we discuss our computations we pause and explain how computations of the unitary nilpotent group give explicit computations of the $L$-theory of the infinite dihedral group. We rely on the Mayer-Vietoris exact sequence (see Cappell [7):

$$
\begin{aligned}
\cdots \rightarrow L_{n}(\mathbb{Z}) \rightarrow L_{n}\left(\mathbb{Z}\left[\mathbb{Z}_{2}\right], w_{1}\right) \oplus L_{n}\left(\mathbb{Z}\left[\mathbb{Z}_{2}\right], w_{2}\right) \\
\rightarrow \frac{L_{n}\left(\mathbb{Z}\left[D_{\infty}\right], w\right)}{\mathrm{UNil}_{n}\left(\mathbb{Z} ; \mathbb{Z}^{\varepsilon_{1}}, \mathbb{Z}^{\varepsilon_{2}}\right)} \rightarrow L_{n-1}(\mathbb{Z}) \rightarrow \cdots
\end{aligned}
$$

where $w_{i}=\left.w\right|_{\left\langle a_{i}\right\rangle}$ and $\varepsilon_{i}=w\left(a_{i}\right)= \pm 1$. We assume $\varepsilon_{2} \geq \varepsilon_{1}$ and write $\widetilde{L}_{n}(\mathbb{Z}[G], w)$ for the cokernel of the natural map $L_{n}(\mathbb{Z}) \rightarrow L_{n}(\mathbb{Z}[G], w)$. The above sequence, and the calculational results in Wall [29, Chapter 13A], quickly lead us to the following equations.

$$
\left.L_{n}\left(\mathbb{Z}\left[D_{\infty}\right], w\right)=\widetilde{L}_{n}\left(\mathbb{Z}_{\mathbb{Z}_{2}}\right], w_{1}\right) \oplus L_{n}\left(\mathbb{Z}\left[\mathbb{Z}_{2}\right], w_{2}\right) \oplus \operatorname{UNil}_{n}\left(\mathbb{Z} ; \mathbb{Z}^{\varepsilon_{1}}, \mathbb{Z}^{\varepsilon_{2}}\right)
$$

unless $n \equiv 1 \bmod 4$, and $w_{1}, w_{2}$ are both nontrivial; in this case, we get:

$$
L_{1}\left(\mathbb{Z}\left[D_{\infty}\right], w\right)=\mathrm{UNil}_{1}\left(\mathbb{Z} ; \mathbb{Z}^{-}, \mathbb{Z}^{-}\right) \oplus L_{0}(\mathbb{Z}) .
$$

For the values of $L_{n}\left(\mathbb{Z}\left[\mathbb{Z}_{2}\right], w\right)$ see Wall [29, Chapter $\left.13 \mathrm{~A}\right]$.

There is another relation between the unitary nilpotent groups and $L$-groups which will be crucial to our computations. Let $R\left[t^{ \pm}\right]$denote the polynomial ring $R[t]$ with + involution $\sum r_{i} t^{i} \mapsto \sum \bar{r}_{i} t^{i}$ or the - involution $\sum r_{i} t^{i} \mapsto$ 
$\sum(-1)^{i} \bar{r}_{i} t^{i}$. Let $\varepsilon_{0}: R[t] \rightarrow R$ be the map of rings given by $\varepsilon_{0}(f(t))=f(0)$; it is a split surjection with the splitting $s_{0}: R \rightarrow R[t]$ given by constant polynomials. Define

$$
N^{ \pm} L_{n}(R)=\operatorname{ker}\left(\varepsilon_{0}: L_{n}\left(R\left[t^{ \pm}\right]\right) \rightarrow L_{n}(R)\right) .
$$

Then $L_{n}\left(R\left[t^{ \pm}\right]\right)=L_{n}(R) \oplus N^{ \pm} L_{n}(R)$. Note that $L_{n}(\mathbb{Z}) \cong \mathbb{Z}, 0, \mathbb{Z}_{2}, 0$ for $n \equiv$ $0,1,2,3(\bmod 4)$ so our computation of $N^{ \pm} L_{n}(\mathbb{Z})$ given below also computes $L_{n}\left(\mathbb{Z}\left[t^{ \pm}\right]\right)$.

Theorem 1.1 (Connolly-Ranicki) There is an isomorphism, natural in $R$,

$$
r: \mathrm{UNil}_{n}\left(R ; R^{ \pm}, R\right) \rightarrow N^{ \pm} L_{n}(R)
$$

Connolly-Ranicki [1], Theorem A] prove this in the + case; we remark here that their formula [11, Definition 2.13] for $r$ and their proof that $r$ is an isomorphism apply equally well in the - case. Note that this implies a 2 -fold periodicity

$$
N^{-} L_{n}(R) \cong \operatorname{UNil}_{n}\left(R ; R^{-}, R\right) \cong \operatorname{UNil}_{n+2}\left(R ; R, R^{-}\right) \cong N^{-} L_{n+2}(R) .
$$

These $N L$-groups are analogous to Bass' nilpotent $K$-groups $N K_{i}(R)=$ $\operatorname{ker}\left(\varepsilon: K_{i}\left(R[t] \rightarrow K_{i}(R)\right)\right.$ occurring in the fundamental theorem of algebraic $K$-theory [2].

The following theorem, which is an easy consequence of Theorem 4.6. provides the calculation of $N^{ \pm} L_{2 k}(\mathbb{Z})$.

Theorem 1.2 There are isomorphisms of abelian groups:

(1) $\varepsilon_{0 *}: L_{0}(\mathbb{Z}[t]) \stackrel{\cong}{\longrightarrow} L_{0}(\mathbb{Z})$. Thus $N L_{0}(\mathbb{Z})=0$.

(2) $\quad P_{2}^{\mathbb{Z}[t]}: t \mathbb{Z}_{2}[t] /\left\{p^{2}-p: p \in t \mathbb{Z}_{2}[t]\right\} \stackrel{\cong}{\longrightarrow} N L_{2}(\mathbb{Z})$.

(3) $P_{2}^{\mathbb{Z}\left[t^{-}\right]}: t^{2} \mathbb{Z}_{2}\left[t^{2}\right] /\left\{p^{2}-p: p \in t^{2} \mathbb{Z}_{2}\left[t^{2}\right]\right\} \stackrel{\cong}{\rightrightarrows} N^{-} L_{2}(\mathbb{Z})$.

(4) $Q_{0}^{\mathbb{Z}\left[t^{-}\right]}: t^{2} \mathbb{Z}_{2}\left[t^{2}\right] /\left\{p^{2}-p: p \in t^{2} \mathbb{Z}_{2}\left[t^{2}\right]\right\} \stackrel{\cong}{\rightrightarrows} N^{-} L_{0}(\mathbb{Z})$.

The maps $P_{2}^{\mathbb{Z}\left[t^{ \pm}\right]}$and $Q_{0}^{\mathbb{Z}\left[t^{-}\right]}$are defined in Section 4 . The inverse maps in (2), (3), and (4) are all essentially given by the Arf invariant of the function field $\mathbb{Z}_{2}(t)$. Also in Section 4, we compute $L_{2}\left(\mathbb{Z}_{2}[t]\right)=L_{0}\left(\mathbb{Z}_{2}[t]\right)$ and show it is isomorphic to $L_{2}(\mathbb{Z}[t])$.

But why do these polynomials $p(t)$ appear in the computation of the $L$-groups? None of the groups above are $\mathbb{Z}[t]$-modules, but the above isomorphisms seem to be more than isomorphisms of abelian groups. We explain that now. 
For each integer $i>0$, and each ring $R$, we have a ring endomorphism:

$$
V_{i}: R[t] \rightarrow R[t] \quad V_{i}(p(t))=p\left(t^{i}\right) .
$$

Note $\varepsilon_{0} \circ V_{i}=\varepsilon_{0}$. The resulting monoid of endomorphisms of $L_{n}(\mathbb{Z}[t])$,

$$
\mathcal{M}=\left\{V_{1}, V_{2}, V_{3}, \ldots\right\} ; \quad V_{i} V_{j}=V_{i j}
$$

therefore makes $L_{n}(\mathbb{Z}[t])$ and $N L_{n}(\mathbb{Z})$ modules over the Verschiebung Algebra,

$$
\mathcal{V}=\mathbb{Z}[\mathcal{M}]
$$

a polynomial ring on $\left\{V_{p}: p\right.$ is a prime $\}: \mathcal{V}=\mathbb{Z}\left[V_{2}, V_{3}, V_{5}, \ldots\right]$. The subalgebra indexed by the odd primes, $\mathcal{V}_{\text {od }}:=\mathbb{Z}\left[V_{3}, V_{5}, V_{7}, V_{11}, \ldots\right]$ acts similarly on $N^{-} L_{n}(\mathbb{Z})$. (Note that for $i>0$ even, $V_{i}: \mathbb{Z}\left[t^{-}\right] \rightarrow \mathbb{Z}\left[t^{-}\right]$is not a map of rings with involution.) The map in Theorem $1.2(2)$ is a map of $\mathcal{V}$-modules and the maps in Theorem 1.2(3) and 1.2(4) are maps of $\mathcal{V}_{\mathrm{od}}$-modules. We have the following reformulation of Theorem 1.2 .

\section{Theorem 1.3}

$$
\begin{aligned}
& N L_{2 k}\left(\mathbb{Z}_{2}\right) \cong N L_{2}(\mathbb{Z}) \cong \mathcal{V} /\left\langle 2, V_{2}-1\right\rangle \\
& N^{-} L_{2}(\mathbb{Z}) \cong N^{-} L_{0}(\mathbb{Z}) \cong \mathcal{V}_{\text {od }} /\langle 2\rangle \\
& N L_{0}(\mathbb{Z})=0
\end{aligned}
$$

To attack the odd-dimensional $L$ - and UNil-groups, we use the classical technique of quadratic linking forms. Given a ring with involution $R$ and a central multiplicative subset $S=\bar{S}$ of non-zero divisors, one can define $L_{2 k}(R, S)$ to be the Witt group of $(-1)^{k}$-quadratic linking forms on finitely generated $S$-torsion $R$-modules of length one (see [21]). Furthermore, one can identify $L_{2 k}(R, S)$ with the relative $L$-group $L_{2 k}\left(R \rightarrow S^{-1} R\right)$. There is an analogous theory for $L_{2 k+1}$.

For a ring with involution $R$ where 2 is not a divisor of zero, define $\langle 2\rangle=\left\{2^{i}\right.$ : $i \geq 0\} \subset R$ and

$$
\left.N^{ \pm} L_{n}(R,\langle 2\rangle)=\operatorname{ker} \varepsilon_{0}: L_{n}\left(R\left[t^{ \pm}\right]\right),\langle 2\rangle\right) \rightarrow L_{n}(R,\langle 2\rangle) .
$$

Proposition 1.4 For any Dedekind domain with involution $R$ where 2 is not a divisor of zero,

$$
N^{ \pm} L_{n}(R,\langle 2\rangle) \cong N^{ \pm} L_{n-1}(R)
$$


Proof By comparing the long exact sequence of the ring map

$$
R\left[t^{ \pm}\right] \rightarrow R[1 / 2]\left[t^{ \pm}\right]
$$

with that of $R \rightarrow R[1 / 2]$, one obtains a localization exact sequence

$$
\cdots \rightarrow N^{ \pm} L_{n}(R[1 / 2]) \rightarrow N^{ \pm} L_{n}(R,\langle 2\rangle) \rightarrow N^{ \pm} L_{n-1}(R) \rightarrow N^{ \pm} L_{n-1}(R[1 / 2]) \cdots
$$

Since 2 is a unit, $N^{ \pm} L_{k}(R[1 / 2])=N^{ \pm} L^{k}(R[1 / 2])$. Connolly-Ranicki show that for a Dedekind domain $\Lambda$ with involution, $N^{ \pm} L^{k}(\Lambda)=0$ (see 11, Proposition 2.11, Proposition 2.19, and the discussion after Proposition 2.19]).

These Witt groups of quadratic linking forms are the main object of study in our paper. They occur so often that introduce new notation for them.

$$
\begin{aligned}
\mathcal{L}\left(\mathbb{Z}\left[t^{ \pm}\right],\langle 2\rangle\right) & =L_{0}\left(\mathbb{Z}\left[t^{ \pm}\right],\langle 2\rangle\right) \\
\widetilde{\mathcal{L}}\left(\mathbb{Z}\left[t^{ \pm}\right],\langle 2\rangle\right) & =N^{ \pm} L_{0}(\mathbb{Z},\langle 2\rangle) \\
\mathcal{L}(\mathbb{Z},\langle 2\rangle) & =L_{0}(\mathbb{Z},\langle 2\rangle) \cong \mathbb{Z}_{2} \oplus \mathbb{Z}_{8} \text { (see [24, Theorem 5.2.2]) }
\end{aligned}
$$

We also use the notation $\mathcal{L}\left(\mathbb{Z}\left[t^{ \pm}\right], 2^{n}\right)$ to denote the Witt group of quadratic linking forms on length one $\mathbb{Z}[t]$-modules of exponent $2^{n}$ (see Section 2, ) To study these groups we use the method of characteristic elements.

A key technical result for us in the following devissage result (see Section 5).

Theorem $1.5 \quad \widetilde{\mathcal{L}}\left(\mathbb{Z}\left[t^{ \pm}\right], 2\right) \cong \widetilde{\mathcal{L}}\left(\mathbb{Z}\left[t^{ \pm}\right],\langle 2\rangle\right)$.

We next present some examples of quadratic linking forms on $\mathbb{Z}[t]$-modules with exponent 2 .

Definition 1.6 For polynomials $p, g \in \mathbb{Z}[t]$, define the quadratic linking form

$$
\mathcal{N}_{p, g}=\left(\mathbb{Z}_{2}[t]^{2},\left(\begin{array}{cc}
p / 2 & 1 / 2 \\
1 / 2 & 0
\end{array}\right),\left(\begin{array}{c}
p / 2 \\
g
\end{array}\right)\right)
$$

By this notation we mean that if $\left\{e_{1}, e_{2}\right\}$ is the standard basis of $\mathbb{Z}_{2}[t]^{2}$ over $\mathbb{Z}_{2}[t]$, then the $2 \times 2$ matrix is $\left(b\left(e_{i}, e_{j}\right)\right)$ and the column vector is $\left(q\left(e_{i}\right)\right)$. If $p(0) \in 4 \mathbb{Z}$, or $g(0) \in 2 \mathbb{Z}$, then $\left[\mathcal{N}_{p, g}\right]$ is an element of $\widetilde{\mathcal{L}}(\mathbb{Z}[t],\langle 2\rangle)$.

We wish compute these Witt groups as modules over the Verschiebung Algebra. To this end, note $t \mathbb{Z}_{2}[t]$ is a $\mathcal{V}$-module in the obvious way: $V_{n}(p(t))=p\left(t^{n}\right)$. 
But, as a less obvious module, $\left[t \mathbb{Z}_{2}[t]\right]$ shall denote the abelian group $t \mathbb{Z}_{2}[t]$, equipped with the following $\mathcal{V}$-module structure:

$$
\begin{aligned}
V_{2 n+1}(p(t)) & =p\left(t^{2 n+1}\right) ; \\
V_{2 n}(p(t)) & =0,
\end{aligned}
$$

for all $n \geq 0$, and all $p \in t \mathbb{Z}_{2}[t]$.

We are now is a position to state our main theorem. (See Section 6 for the proof.)

Theorem 1.7 (1) There is an isomorphism of $\mathcal{V}$-modules,

$$
j_{1}+j_{2}: \frac{t \mathbb{Z}_{4}[t]}{\left(2 V_{2}-2\right) t \mathbb{Z}_{4}[t]} \oplus\left[t \mathbb{Z}_{2}[t]\right] \rightarrow \widetilde{\mathcal{L}}(\mathbb{Z}[t],\langle 2\rangle)
$$

given by $j_{1}[t p]=\left[\mathcal{N}_{t p, 1}\right]$ and $j_{2}[t p]=\left[\mathcal{N}_{1, t p}\right]-\left[\mathcal{N}_{t, p}\right]$.

(2) There is an isomorphism of $\mathcal{V}_{\text {od }}$-modules,

$$
j: t^{2} \mathbb{Z}_{2}\left[t^{2}\right] \rightarrow \widetilde{\mathcal{L}}\left(\mathbb{Z}\left[t^{-}\right],\langle 2\rangle\right) ; \quad j\left(t^{2} p\left(t^{2}\right)\right)=\left[\mathcal{N}_{t^{2} p\left(t^{2}\right), 1}\right] .
$$

\section{Corollary 1.8}

$$
\widetilde{\mathcal{L}}(\mathbb{Z}[t],\langle 2\rangle)=\bigoplus_{k=-1}^{\infty} \mathcal{V} \cdot b_{k} ; \quad \widetilde{\mathcal{L}}\left(\mathbb{Z}\left[t^{-}\right],\langle 2\rangle\right)=\bigoplus_{j=1}^{\infty} \mathcal{V}_{\text {od }} \cdot c_{j}
$$

where $b_{-1}=\left[\mathcal{N}_{t, 1}\right]$ and $b_{k}=\left[\mathcal{N}_{1, t^{2}}\right]-\left[\mathcal{N}_{t, t^{2^{k}-1}}\right]$ for all $k \geq 0$, and $c_{j}=\mathcal{N}_{t^{2}, 1}$, for all $j \geq 1$. The annihilator ideals of these elements are:

$$
\operatorname{Ann}\left(b_{-1}\right)=\left\langle 4,2 V_{2}-2\right\rangle ; \operatorname{Ann}\left(b_{k}\right)=\left\langle 2, V_{2}\right\rangle \text { for } k \geq 0 ; \operatorname{Ann}_{\mathcal{V}_{\text {od }}}\left(c_{j}\right)=\langle 2\rangle .
$$

Proof Note that $t \mathbb{Z}[t]$ is a free rank one $\mathcal{V}$-module. In $\frac{t \mathbb{Z}_{4}[t]}{\left(2 V_{2}-2\right) t \mathbb{Z}_{4}[t]}$, a $\mathcal{V}$ generator is $t$, with annihilator ideal $\left\langle 4,2 V_{2}-2\right\rangle$. Also $\left[t \mathbb{Z}_{2}[t]\right]$ is a free module over $\mathcal{V} /\left\langle 2, V_{2}\right\rangle$, with basis $\left\{t^{2^{k}}: k=0,1,2, \ldots\right\}$. Finally the set $\left\{t^{2^{j}}: j=\right.$ $1,2,3, \ldots\}$ is a $\mathcal{V}_{\text {od }} / 2 \mathcal{V}_{\text {od }}$ basis for $t^{2} \mathbb{Z}\left[t^{2}\right]$. The result now follows from Theorem 1.7.

As a consequence of our computation we have the following corollary (cf [22]).

Corollary 1.9 There is an element $\alpha \in \mathrm{UNil}_{3}(\mathbb{Z} ; \mathbb{Z}, \mathbb{Z})$ of order 4.

We give a separate, elementary proof of this using Gauss sums in Section 3 .

The heart of our paper is the following set of calculations which we summarize as follows: 


\section{Theorem 1.10}

$$
\begin{aligned}
& \mathrm{UNil}_{0}\left(\mathbb{Z} ; \mathbb{Z}^{-}, \mathbb{Z}\right)=\mathrm{UNil}_{2}\left(\mathbb{Z} ; \mathbb{Z}^{-}, \mathbb{Z}\right) \cong \mathcal{V}_{\text {od }} /\langle 2\rangle \quad(\text { Theorem } 4.6) \\
& \mathrm{UNil}_{1}\left(\mathbb{Z} ; \mathbb{Z}^{-}, \mathbb{Z}\right)=\mathrm{UNil}_{3}\left(\mathbb{Z} ; \mathbb{Z}^{-}, \mathbb{Z}\right) \cong \bigoplus_{i=1}^{\infty} \mathcal{V}_{\text {od }} /\langle 2\rangle \quad \text { (Corollary [1.8) } \\
& \mathrm{UNil}_{0}(\mathbb{Z} ; \mathbb{Z}, \mathbb{Z})=0 \quad \text { (Theorem 4.6) } \\
& \mathrm{UNil}_{1}(\mathbb{Z} ; \mathbb{Z}, \mathbb{Z})=0 \quad \text { (Proposition 6.3) } \\
& \mathrm{UNil}_{2}(\mathbb{Z} ; \mathbb{Z}, \mathbb{Z}) \cong \mathcal{V} /\left\langle 2, V_{2}-1\right\rangle \quad \text { (Theorem 4.6) } \\
& \mathrm{UNil}_{3}(\mathbb{Z} ; \mathbb{Z}, \mathbb{Z}) \cong \mathcal{V} /\left\langle 4,2 V_{2}-2\right\rangle \oplus \bigoplus_{i=0}^{\infty} \mathcal{V} /\left\langle 2, V_{2}\right\rangle \quad \text { (Corollary 1.8) }
\end{aligned}
$$

Finally, let $\mathbb{Z}\left[t, t^{-1}\right]^{ \pm}$be the Laurent polynomial ring $\mathbb{Z}\left[t, t^{-1}\right]$ with the involution $t \mapsto \pm t$. The following formulas and the results of this paper compute $L_{n}\left(\mathbb{Z}\left[t, t^{-1}\right]^{ \pm}\right)$.

$$
\begin{aligned}
L_{n}\left(\mathbb{Z}\left[t, t^{-1}\right]\right) & \cong L_{n}(\mathbb{Z})^{2} \oplus N L_{n}(\mathbb{Z})^{2} \\
L_{n}\left(\mathbb{Z}\left[t, t^{-1}\right]^{-}\right) & \cong L_{n}(\mathbb{Z}) \oplus L_{n+2}(\mathbb{Z}) \oplus N^{-} L_{n}(\mathbb{Z})^{2}
\end{aligned}
$$

The first formula is proved by Ranicki in [19] in the oriented case, and the second can be proven with similar techniques.

\section{Definitions}

Here we present a unified framework including both the Witt group of quadratic linking forms over a ring $R$, and the surgery obstruction groups $L_{2 k}(R)$.

Let $R$ be a ring. An $R$-module $M$ has length one if there is a short exact sequence $0 \rightarrow F_{1} \rightarrow F_{0} \rightarrow M \rightarrow 0$, where $F_{0}$ and $F_{1}$ are finitely generated free $R$-modules. A submodule $L$ of a length one module $M$ has colength one if $M / L$ has length one. This implies $L$ has length one.

A ring with involution is a ring $R$ with a function $-: R \rightarrow R$ satisfying $\overline{1}=1$, $\overline{\bar{r}}=r, \overline{r+s}=r+s$, and $\overline{r s}=\bar{s} \bar{r}$ for all $r, s \in R$. An $R$-bimodule with involution is an $R$-bimodule $A$ with $\mathbb{Z}$-automorphism - : $A \rightarrow A$ of order 2 satisfying $\overline{r a s}=\bar{s} \bar{a} \bar{r}$ for all $r, s \in R, a \in A$.

Let $(R, A)$ be a ring $R$ with involution, together with some $R$-bimodule with involution. If $M$ is a left $R$-module, then $M^{\wedge}:=\operatorname{Hom}_{R}(M, A)$ is also a left 
$R$-module if we set $(r \varphi)(m)=\varphi(m) \bar{r}$. A symmetric form over $(R, A)$ is an $R$-module $M$ and a function $\lambda: M \times M \rightarrow A$ satisfying:

$$
\begin{aligned}
\lambda(x, y) & =\overline{\lambda(y, x)} \\
\lambda\left(x, y+y^{\prime}\right) & =\lambda(x, y)+\lambda\left(x, y^{\prime}\right) \\
\lambda(x, r y) & =r \lambda(x, y)
\end{aligned}
$$

for all $r \in R, x, y, y^{\prime} \in M$. If the $R$-map

$$
\operatorname{Ad} \lambda: M \rightarrow M^{\wedge}=\operatorname{Hom}_{R}(M, A) \quad x \mapsto(y \mapsto \lambda(x, y))
$$

is an isomorphism, then $(M, \lambda)$ is nonsingular.

A (nonsingular) quadratic form over $(R, A)$ is a (nonsingular) symmetric form $(M, \lambda)$ over $(R, A)$ and a function $\mu: M \rightarrow A /\{a-\bar{a}: a \in A\}$ so that

$$
\begin{aligned}
\lambda(x, x) & =\{1+*\} \mu(x) \\
{[\lambda(x, y)] } & =\mu(x+y)-\mu(x)-\mu(y) \\
\mu(r x) & =r \mu(x) \bar{r}
\end{aligned}
$$

for all $r \in R, x, y \in M$. Here [ ]: $A \rightarrow A /\{a-\bar{a}: a \in A\}, a \mapsto[a]$ is the quotient map and $\{1+*\}: A /\{a-\bar{a}: a \in A\} \rightarrow A$ is given by $[a] \mapsto a+\bar{a}$.

A skew-quadratic (or $(-1)$-quadratic) form over $(R, A)$ is a quadratic form over $\left(R, A^{-}\right)$. Here $A^{-}$is the bimodule $A$, but with the involution: $a \mapsto-\bar{a}$.

Definition 2.1 Suppose $R$ is a ring with involution, $A$ is an $R$-bimodule with involution, and $\mathbf{M}$ is a class of left $R$-modules. We assume $(R, A, \mathbf{M})$ satisfies the following properties:

(1) If $M \in \mathbf{M}$, then $M^{\wedge} \in \mathbf{M}$, and the double duality map $M \stackrel{D}{\rightarrow} M^{\wedge \wedge}$ is an isomorphism.

(2) The direct sum of two modules in $\mathbf{M}$ is in $\mathbf{M} .\{0\}$ is in $\mathbf{M}$.

(3) If $0 \rightarrow M_{1} \rightarrow M_{2} \rightarrow M_{3} \rightarrow 0$ is exact and $M_{2}, M_{3}$ are in $\mathbf{M}$, then $M_{1}$ is also in $\mathbf{M}$, and $0 \rightarrow M_{3}^{\wedge} \rightarrow M_{2}^{\wedge} \rightarrow M_{1}^{\wedge} \rightarrow 0$ is also exact.

A $(R, A, \mathbf{M})$-form is a nonsingular quadratic form $m=(M, \lambda, \mu)$ over $(R, A)$, such that $M \in \mathbf{M}$.

Next we give the examples we care about. It is clear that the first example below satisfies the conditions of 2.1. Proposition A.1 proves that the second example also satisfies the conditions.

- $(R, R, \mathbf{F})$. Here $R$ is a ring with involution; $A=R ; \mathbf{F}$ is the class of all finitely generated stably free $R$-modules. We will also use $\left(R, R^{-}, \mathbf{F}\right)$. 
- $\left(R, \mathbb{Q} R / R, \mathbf{M}_{2^{n}}\right)$. Here $R$ is a ring with involution which is torsion free as an abelian group and $A=\mathbb{Q} R / R$, where $\mathbb{Q} R$ denotes the localization of $R$ obtained by inverting the positive integers in $R ; \mathbf{M}_{2^{n}}$ is the class of all length one $R$-modules $M$ for which $2^{n} M=0$.

We will also use $(R, \mathbb{Q} R / R, \mathbf{M}\langle 2\rangle)$, where $\mathbf{M}\langle 2\rangle:=\cup_{n \geq 0} \mathbf{M}_{2^{n}}$.

A subLagrangian for a $(R, A, \mathbf{M})$-form $m=(M, \lambda, \mu)$ is a submodule $L \subseteq M$ such that $M / L \in \mathbf{M}, \mu(L)=0$, and $L \subseteq L^{\perp}$, where

$$
L^{\perp}:=\{x \in M: \lambda(x, L)=0\} .
$$

If $L=L^{\perp}$, we say $L$ is a Lagrangian for $m$, and that $m$ admits a Lagrangian.

Define an equivalence relation on the collection of $(R, A, \mathbf{M})$-forms by:

$$
m \sim m^{\prime} \quad \text { if } \quad m \oplus m_{0} \cong m^{\prime} \oplus m_{0}^{\prime}
$$

where $m_{0}$ and $m_{0}^{\prime}$ are $(R, A, \mathbf{M})$-forms which admit Lagrangians. (Here $\cong$ means "is isometric to".)

Definition 2.2 The quadratic Witt group $Q W(R, A, \mathbf{M})$ is the abelian group of equivalence classes of $(R, A, \mathbf{M})$-forms. Addition is orthogonal direct sum.

The negative of $[M, \lambda, \mu]$ is $[M,-\lambda,-\mu]$, since their sum admits the diagonal Lagrangian $\{(x, x): x \in M\}$.

The SubLagrangian Construction Given a $(R, A, \mathbf{M})$-form $m=(M, \lambda, \mu)$ and a subLagrangian $S$ for $m$, we define an induced quadratic form

$$
m_{S}=\left(S^{\perp} / S, b_{S}, \mu_{S}\right): \quad b_{S}([x],[y])=b(x, y) ; \quad \mu_{S}([x])=\mu(x) .
$$

Lemma A.2 shows that $m_{S}$ is again a $(R, A, \mathbf{M})$-form and $[m]=\left[m_{S}\right] \in$ $Q W(R, A, \mathbf{M})$.

The Wall surgery obstruction groups of $R$ (in even dimensions) are:

$$
L_{0}(R)=Q W(R, R, \mathbf{F}) \quad L_{2}(R)=Q W\left(R, R^{-}, \mathbf{F}\right),
$$

where $\mathbf{F}$ is the class of finitely generated, stably free $R$-modules.

We are going to define the Witt groups of quadratic linking forms $\mathcal{L}\left(R, 2^{n}\right)$ and $\mathcal{L}(R,\langle 2\rangle)$, so that,

$$
\begin{aligned}
\mathcal{L}\left(R, 2^{n}\right) & \cong Q W\left(R, \mathbb{Q} R / R, \mathbf{M}_{2^{n}}\right) \\
\mathcal{L}(R,\langle 2\rangle) & \cong Q W(R, \mathbb{Q} R / R, \mathbf{M}\langle 2\rangle)
\end{aligned}
$$

But for historical reasons (see for example [28]), we will first change coordinates, and do all of our work in the classical regime of quadratic linking forms. 
Definition 2.3 Assume $R$ is a ring with involution which is torsion free as an abelian group.

A quadratic linking form over $R$ is a triple $(M, b, q)$ such that $M \in \mathbf{M}_{2^{n}}$ for some $n,(M, b)$ is a nonsingular symmetric form over $(R, \mathbb{Q} R / R)$, and $q: M \rightarrow$ $\mathbb{Q} R /(1+*) R$ is a function satisfying:

$$
\begin{aligned}
{[q(x)] } & =b(x, x) \in \mathbb{Q} R / R, \\
q(x+y)-q(x)-q(y) & =\{1+*\} b(x, y) \in \mathbb{Q} R /(1+*) R, \\
q(r x) & =r q(x) \bar{r} .
\end{aligned}
$$

Here

$$
\{1+*\}: \frac{\mathbb{Q} R / R}{(1-*) \mathbb{Q} R / R} \rightarrow \frac{(1+*) \mathbb{Q} R}{(1+*) R}
$$

is the isomorphism: $[x] \mapsto(x+\bar{x}) \bmod (1+*) R$.

Note $2^{n+1} q(x)=0$, whenever $2^{n} b(x, x)=0$. Also $q(M) \subset \frac{(1+*) \mathbb{Q} R}{(1+*) R}$.

In other words, a quadratic linking form $(M, b, q)$ is any triple which can be written in the form $(M, b,\{1+*\} \mu)$, where $(M, b, \mu)$ is a $(R, \mathbb{Q} R / R, \mathbf{M}\langle 2\rangle)$ form.

Let $\Lambda\left(R, 2^{n}\right)$ denote the set of isometry classes of quadratic linking forms with exponent $2^{n}$. The rule

$$
(M, \lambda, \mu) \leftrightarrow(M, \lambda,\{1+*\} \mu)
$$

gives a one to one correspondence between the set of isometry classes of $\left(R, \mathbb{Q} R / R, \mathbf{M}_{2^{n}}\right)$-forms and $\Lambda\left(R, 2^{n}\right)$.

A Lagrangian for a quadratic linking form $(M, b,\{1+*\} \mu)$ is defined to be a Lagrangian for the $(R, \mathbb{Q} R / R, \mathbf{M}\langle 2\rangle)$-form $(M, b, \mu)$.

Two elements $m, m^{\prime} \in \Lambda\left(R, 2^{n}\right)$ are equivalent if $m \oplus m_{0} \cong m^{\prime} \oplus m_{0}^{\prime}$ where $m_{0}$ and $m_{0}^{\prime}$ are elements of $\Lambda\left(R, 2^{n}\right)$ which admit Lagrangians.

$$
\text { Set: } \quad \mathcal{L}\left(R, 2^{n}\right)=\Lambda\left(R, 2^{n}\right) / \sim,
$$

an abelian group under orthogonal direct sum. (3) induces an isomorphism

$$
Q W\left(R, \mathbb{Q} R / R, \mathbf{M}_{2^{n}}\right) \cong \mathcal{L}\left(R, 2^{n}\right) .
$$

Similarly, the above equivalence relation on each of the $\Lambda\left(R, 2^{n}\right)$ 's defines an equivalence relation on their union,

$$
\Lambda(R,\langle 2\rangle)=\cup_{n=1}^{\infty} \Lambda\left(R, 2^{n}\right),
$$


and the abelian group of equivalence classes,

$$
\mathcal{L}(R,\langle 2\rangle):=\Lambda(R,\langle 2\rangle) / \sim
$$

is canonically isomorphic to $Q W(R, \mathbb{Q} R / R, \mathbf{M}\langle 2\rangle)$ by the rule (3).

One could define a corresponding group of skew-quadratic linking forms, but we do not do so here, because we show in Proposition 6.3 that when $R=\mathbb{Z}$, any such form admits a Lagrangian.

\section{The proof of Corollary 1.9}

In this section we give a short proof, independent of the rest of this paper, of the fact that $\widetilde{\mathcal{L}}(\mathbb{Z}[t], 2) \cong \operatorname{UNil}_{3}(\mathbb{Z} ; \mathbb{Z}, \mathbb{Z}) \cong L_{3}(\mathbb{Z}[t])$ has an element of order 4 . We first review the isomorphism [24, 5.2.2].

$$
\operatorname{Rk} \oplus \mathrm{GS}: \mathcal{L}(\mathbb{Z},\langle 2\rangle) \stackrel{\simeq}{\longrightarrow} \mathbb{Z}_{2} \oplus \mathbb{Z}_{8}
$$

The rank homomorphism is

$$
\operatorname{Rk}[M, b, q]= \begin{cases}0 & \text { if }|M|=2^{2 k} \\ 1 & \text { if }|M|=2^{2 k+1}\end{cases}
$$

The Gauss sum homomorphism is

$$
\operatorname{GS}[M, b, q]=[k] \in \mathbb{Z}_{8}
$$

where

$$
\frac{1}{\sqrt{|M|}} \sum_{x \in M} e^{\pi i q(x)}=e^{2 \pi i k} .
$$

Now let $\alpha=\left[\mathbb{Z}_{2}[t]^{2},\left(\begin{array}{cc}1 / 2 & 0 \\ 0 & 1 / 2\end{array}\right),\left(\begin{array}{c}1 / 2 \\ t-1 / 2\end{array}\right)\right] \in \mathcal{L}(\mathbb{Z}[t],\langle 2\rangle)$. Note that $\varepsilon_{0}(\alpha)$ has the diagonal Lagrangian $L=\{(0,0),(1,1)\}$, so $\alpha \in \widetilde{\mathcal{L}}(\mathbb{Z}[t],\langle 2\rangle)$. Consider now the ring map

$$
\varepsilon_{1}: \mathbb{Z}[t] \rightarrow \mathbb{Z}, \quad f(t) \mapsto f(1) .
$$

Then: $\quad(\mathrm{Rk} \oplus \mathrm{GS}) \varepsilon_{1}(\alpha)=0 \oplus 2 \in \mathbb{Z}_{2} \oplus \mathbb{Z}_{8}$,

so $\alpha \in \widetilde{\mathcal{L}}(\mathbb{Z}[t],\langle 2\rangle)$ is an element of order at least 4 . One can show $4 \alpha=0$ by quoting Farrell's Exponent Four Theorem [12] or by showing directly that $4 \alpha$ has a Lagrangian. 


\section{Computation of $\mathrm{UNil}_{2 n}(\mathbb{Z} ; \mathbb{Z}, \mathbb{Z}), \mathrm{UNil}_{2 n}\left(\mathbb{Z} ; \mathbb{Z}^{-}, \mathbb{Z}\right)$, and $\mathcal{L}^{\mathrm{ev}}\left(\mathbb{Z}\left[t^{ \pm}\right], 2\right)$}

According to Theorem 1.1

$$
L_{n}\left(\mathbb{Z}\left[t^{ \pm}\right]\right)=L_{n}(\mathbb{Z}) \oplus N^{ \pm} L_{n}(\mathbb{Z})=L_{n}(\mathbb{Z}) \oplus \operatorname{UNil}_{n}\left(\mathbb{Z} ; \mathbb{Z}^{ \pm}, \mathbb{Z}\right) .
$$

In this section we compute $L_{2 n}\left(\mathbb{Z}\left[t^{ \pm}\right]\right)$, and therefore $\operatorname{UNil}_{2 n}\left(\mathbb{Z} ; \mathbb{Z}^{ \pm}, \mathbb{Z}\right)$. We also compute the group $\mathcal{L}^{\mathrm{ev}}\left(\mathbb{Z}\left[t^{ \pm}\right], 2\right)$ of quadratic linking forms of exponent 2 with even type, as defined below.

Throughout this section, $R$ denotes a ring with involution.

Definition 4.1 Fix an integer $n>0$. Assume $R$ is torsion free as an abelian group. Let $\Lambda^{\mathrm{ev}}\left(R, 2^{n}\right)$ be the collection of all quadratic linking forms $m=$ $(M, b, q)$ for which $2^{n} M=0$ and $2^{n} q(x)=0$ for all $x \in M$. Quadratic linking forms in $\Lambda^{\mathrm{ev}}\left(R, 2^{n}\right)$ are called forms of exponent $2^{n}$ with even type.

We define an equivalence relation on $\Lambda^{\mathrm{ev}}\left(R, 2^{n}\right)$ by:

$$
m \sim m^{\prime} \Leftrightarrow m \oplus m_{0} \cong m^{\prime} \oplus m_{0}^{\prime}
$$

for some $m_{0}, m_{0}^{\prime}$ in $\Lambda^{\mathrm{ev}}\left(R, 2^{n}\right)$ admitting Lagrangians. The abelian group of equivalence classes of forms of exponent $2^{n}$ with even type is:

$$
\mathcal{L}^{\mathrm{ev}}\left(R, 2^{n}\right)=\Lambda^{\mathrm{ev}}\left(R, 2^{n}\right) / \sim
$$

Forgetful maps define homomorphisms,

$$
\mathcal{L}\left(R, 2^{n-1}\right) \stackrel{j_{n-1}}{\longrightarrow} \mathcal{L}^{\mathrm{ev}}\left(R, 2^{n}\right) \stackrel{\iota_{n}}{\longrightarrow} \mathcal{L}\left(R, 2^{n}\right) \stackrel{i_{n}}{\longrightarrow} \mathcal{L}(R,\langle 2\rangle) .
$$

The map $\iota_{n} \circ j_{n-1}$ is mentioned in Theorem 5.1 below.

There is a natural map

$$
\alpha_{*}: L_{0}(R / 2 R) \rightarrow \mathcal{L}^{\mathrm{ev}}(R, 2), \quad[M, \lambda, \mu] \mapsto[M, \alpha \circ \lambda, \beta \circ \mu]
$$

where

$$
\begin{gathered}
R / 2 R \stackrel{\alpha}{\rightarrow} \mathbb{Q} R / R, \quad[x] \mapsto[x / 2] \\
\frac{R / 2 R}{(1-*)(R / 2 R)} \stackrel{\beta}{\rightarrow} \mathbb{Q} R /(1+*) R, \quad[y] \mapsto[(y+\bar{y}) / 2] .
\end{gathered}
$$

Proposition $4.2 \alpha_{*}: L_{0}\left(\mathbb{Z}_{2}[t]\right) \rightarrow \mathcal{L}^{\mathrm{ev}}(\mathbb{Z}[t], 2)$ is an isomorphism.

Proof $\mathbb{Z}_{2}[t] \stackrel{\alpha}{\rightarrow}{ }_{2}(\mathbb{Q}[t] / \mathbb{Z}[t])$ and $\mathbb{Z}_{2}[t] \stackrel{\beta}{\rightarrow}{ }_{2}(\mathbb{Q}[t] / 2 \mathbb{Z}[t])=\mathbb{Z}_{2}[t]$ are isomorphisms. Here ${ }_{2} A=\{a \in A: 2 a=0\}$ for an abelian group $A$. 
We view

$$
\widehat{H}_{n}\left(\mathbb{Z}_{2} ; R\right)=\frac{\left\{x \in R: \bar{x}=(-1)^{n+1} x\right\}}{\left\{y-(-1)^{n} \bar{y}: y \in R\right\}} .
$$

as a $R / 2 R$-module by the rule $[a] \circ[x]:=[a x \bar{a}]$.

We will need the following three classes of quadratic forms. For each $p, g \in$ $\widehat{H}_{n}\left(\mathbb{Z}_{2} ; R\right)$ (or even $p, g \in R$ representing classes in $\widehat{H}_{n}\left(\mathbb{Z}_{2} ; R\right)$ ), define $\left[P_{p, g}\right] \in$ $L_{2 n}(R)$ by the nonsingular $(-1)^{n}$-quadratic form:

$$
P_{p, g}=\left(R^{2},\left(\begin{array}{cc}
0 & 1 \\
(-1)^{n} & 0
\end{array}\right),\left(\begin{array}{l}
p \\
g
\end{array}\right)\right) .
$$

The notational conventions here are similar to those described after Definition 1.6. The symmetric form $\lambda$ is determined by the matrix $\left(\lambda\left(e_{i}, e_{j}\right)\right)$ and the quadratic refinement $\mu$ is specified by the vector $\left(\mu\left(e_{i}\right)\right)$.

Next assume $p, g \in R$ are in $\operatorname{ker}(1-*)$. Assume $R$ is torsion free as an abelian group. Define $\left[\mathcal{P}_{p, g}\right] \in \mathcal{L}^{\mathrm{ev}}(R, 2)$ and $\left[\mathcal{N}_{p, g}\right] \in \mathcal{L}(R, 2)$ by the quadratic linking forms:

$$
\begin{aligned}
& \mathcal{P}_{p, g}=\left((R / 2 R)^{2},\left(\begin{array}{cc}
0 & 1 / 2 \\
1 / 2 & 0
\end{array}\right),\left(\begin{array}{c}
p \\
g
\end{array}\right)\right) \\
& \mathcal{N}_{p, g}=\left((R / 2 R)^{2},\left(\begin{array}{cc}
p / 2 & 1 / 2 \\
1 / 2 & 0
\end{array}\right),\left(\begin{array}{c}
p / 2 \\
g
\end{array}\right)\right)
\end{aligned}
$$

Note that $\mathcal{N}_{2 p, g}=\mathcal{P}_{p, g}$ and that $\left[P_{p, g}\right]$ maps to $\left[\mathcal{P}_{p, g}\right]$ under the composite

$$
L_{2}(R) \rightarrow L_{2}(R / 2 R)=L_{0}(R / 2 R) \stackrel{\alpha_{*}}{\longrightarrow} \mathcal{L}^{\mathrm{ev}}(R, 2) .
$$

Lemma 4.3 (Formal Properties of $P_{p, g}, \mathcal{P}_{p, g}$, and $\mathcal{N}_{p, g}$ )

(1) $P_{p, g} \cong P_{g, p} ; \quad \mathcal{P}_{p, g} \cong \mathcal{P}_{g, p} \quad$ ( $\cong$ means "is isometric to").

(2) $\left[P_{p_{1}, g} \oplus P_{p_{2}, g}\right]=\left[P_{p_{1}+p_{2}, g}\right] ; \quad\left[\mathcal{N}_{p_{1}, g} \oplus \mathcal{N}_{p_{2}, g}\right]=\left[\mathcal{N}_{p_{1}+p_{2}, g}\right]$.

(3) $2\left[\mathcal{N}_{p, g}\right]=\left[\mathcal{P}_{p, g}\right] ; \quad 2\left[\mathcal{P}_{p, g}\right]=0 ; \quad 2\left[P_{p, g}\right]=0$.

(4) $\left[P_{p g \bar{p}, g}\right]=\left[P_{p, g}\right] ; \quad\left[\mathcal{P}_{p g \bar{p}, g}\right]=\left[\mathcal{P}_{p, g}\right]$.

(5) $\left[\mathcal{N}_{\bar{x} p x, g}\right]=\left[\mathcal{N}_{p, x g} \bar{x}\right] ; \quad\left[P_{\bar{x} p x, g}\right]=\left[P_{p, x g}\right] \quad$ for $x \in R$.

(6) $\left[P_{p, g}\right]=\left[P_{p g, 1}\right]$, if $R=\mathbb{Z}_{2}[t]$, or if $R=\mathbb{Z}[t]$ or $\mathbb{Z}\left[t^{-}\right]$and $n$ is odd.

(7) $V_{k}\left(\left[\mathcal{N}_{p, g}\right]\right)=\left[\mathcal{N}_{p\left(t^{k}\right), g\left(t^{k}\right)}\right]$ in $\mathcal{L}\left(R\left[t^{ \pm}\right], 2\right)$.

(8) $\left[\mathcal{N}_{p, g+h}\right]-\left[\mathcal{N}_{p, g}\right]-\left[\mathcal{N}_{p, h}\right]=\left[\mathcal{P}_{p g \bar{p}, h}\right]$. 
Proof (1) Replace the standard basis $\left\{e_{1}, e_{2}\right\}$ by $\left\{e_{2},(-1)^{n} e_{1}\right\}$.

(2) Let $\left\{e_{1}, e_{2}\right\},\left\{e_{3}, e_{4}\right\}$ be the standard bases for $P_{p_{1}, g}$ and $P_{p_{2}, g}$. A subLagrangian for $m=P_{p_{1}, g} \oplus P_{p_{2}, g}$ is $S=\left\langle e_{2}+e_{4}\right\rangle$. Then $\left\{\left[e_{1}+e_{3}\right],\left[e_{2}\right]\right\}$ is a basis for $S^{\perp} / S$. The subLagrangian construction satisfies $m_{S}=P_{p_{1}+p_{2}, g}$ proving the result. The proof for $\mathcal{N}_{p_{1}, g} \oplus \mathcal{N}_{p_{2}, g}$ is the same.

(3) is immediate from (2) and the definitions, since $2[p / 2]=[p]$ in $\widehat{H}_{1}\left(\mathbb{Z}_{2} ; R\right)$.

(4) By (2) and (3) it suffices to note that $\left\langle e_{1}+p e_{2}\right\rangle$ is a Lagrangian for $P_{p g \bar{p}+p, g}$.

(5) A Lagrangian for $\mathcal{N}_{\bar{x} p x, g} \oplus \mathcal{N}_{-p,-x g \bar{x}}$ is $\left\langle e_{1}+\bar{x} e_{3}, x e_{2}+e_{4}\right\rangle$. The proof of the second part is similar.

(6) We first consider the cases of $R=\mathbb{Z}[t]$ with $n$ odd and $R=\mathbb{Z}_{2}[t]$. By (1) and (2), it is suffices to consider the cases $p=t^{k}, g=t^{l}$, for some $k, l \geq 0$. From (5) above, $\left[P_{t^{k}, t^{l+2 m}}\right]=\left[P_{t^{k+2 m}, t^{l}}\right]$, so we may assume $l$ is 0 or 1 . The case $l=0$ is immediate, so we assume $l=1$ and proceed by induction on $k$. If $k$ is even, the result follows from (5) and (1). If $k=2 i-1$ is odd, then $\left[P_{t^{2 i-1}, t}\right]=\left[P_{t^{i-1}, t}\right]=\left[P_{t^{i}, 1}\right]=\left[P_{t^{2 i}, 1}\right]$; the first equality comes from replacing the standard basis $\left\{e_{1}, e_{2}\right\}$ by $\left\{e_{1}+t^{i-1} e_{2}, e_{2}\right\}$, the second equality from the induction hypothesis, and the third from (4).

The proof in the case $R=\mathbb{Z}\left[t^{-}\right]$and $n$ odd is similar; it involves replacing $t$ by $t^{2}$ everywhere in the above paragraph.

(7) This is immediate from the definitions.

(8) Let $m=\mathcal{N}_{p, g+h} \oplus \mathcal{N}_{-p, g} \oplus \mathcal{N}_{-p, h}$. A subLagrangian for $m$ is $S=\left\langle e_{1}+\right.$ $\left.e_{3}, e_{2}+e_{4}+e_{6}\right\rangle$. An arbitrary element $\sum_{i=1}^{6} a_{i} e_{i} \in(R / 2 R)^{6}$ is in $S^{\perp}$ if and only if: $a_{1} p+a_{2}+a_{3} p+a_{4}=0=a_{1}+a_{3}+a_{5}$. Therefore, $x \in S^{\perp}$ if and only if $x=a_{1}\left(e_{1}+e_{3}\right)+a_{2}\left(e_{2}+e_{4}\right)+a_{5}\left(e_{3}+p e_{4}+e_{5}\right)+a_{6} e_{6}$. Hence $S^{\perp} / S=\left\langle\left[e_{3}+p e_{4}+e_{5}\right],\left[e_{6}\right]\right\rangle$. Thus $[m]=\left[m_{S}\right]=\left[\mathcal{P}_{p g \bar{p}, h}\right]$.

Definition 4.4 Let $R$ be a ring with involution. Define maps

(1) $P_{2}=P_{2}^{R}: \widehat{H}_{1}\left(\mathbb{Z}_{2} ; R\right) \rightarrow L_{2}(R), \quad[p] \mapsto\left[P_{p, 1}\right]$,

$$
Q_{0}=Q_{0}^{R\left[t^{-}\right]}: t^{2} \widehat{H}_{1}\left(\mathbb{Z}_{2} ; R\left[t^{-}\right]\right) \rightarrow N L_{0}^{-}(R), \quad\left[t^{2} p\right] \mapsto\left[P_{t p, t}\right] .
$$

Note that $\varepsilon_{0 *}\left[P_{t p, t}\right]=\left[P_{0,0}\right]=0$, so $Q_{0}$ takes its values in $N^{-} L_{0}(R) \subset$ $L_{0}\left(R\left[t^{-}\right]\right)$. Both $P_{2}$ and $Q_{0}$ are homomorphisms by Lemma 4.3(2).

Lemma 4.5 Let $R$ be a commutative ring with trivial involution.

(1) If $p \in R\left[t^{ \pm}\right]$satisfies $\bar{p}=p$, then $P_{2}^{R\left[t^{ \pm}\right]}\left(\left[p^{2}\right]-[p]\right)=0$. 
(2) If $p \in R\left[t^{-}\right]$satisfies $\bar{p}=p$, then $Q_{0}^{R\left[t^{-}\right]}\left(\left[\left(t^{2} p\right)^{2}\right]-\left[t^{2} p\right]\right)=0$.

(3) $P_{2}^{R[t]}$ is a map of $\mathcal{V}$-modules. $P_{2}^{R\left[t^{-}\right]}$and $Q_{0}^{R\left[t^{-}\right]}$are maps of $\mathcal{V}_{\mathrm{od}^{-}}$ modules. (The Verschiebung algebras $\mathcal{V}$ and $\mathcal{V}_{\text {od }}$ were defined in the introduction.)

Proof Parts (1) and (2) follow from Lemma 4.3(4). The assertions concerning $P_{2}$ in Part (3) are clear. Finally, note

$$
\begin{aligned}
V_{2 k+1} Q_{0}\left[t^{2} p\left(t^{2}\right)\right] & =\left[P_{t^{2 k+1}} p\left(t^{4 k+2}\right), t^{2 k+1}\right] \\
& =\left[P_{t^{4 k+1} p\left(t^{4 k+2}\right), t}\right] \quad \text { by Lemma 4.3 }(5) \\
& =Q_{0} V_{2 k+1}\left[t^{2} p\left(t^{2}\right)\right] .
\end{aligned}
$$

We now specialize to $R=\mathbb{Z}$ and $\mathbb{Z}_{2}$. We will abuse notation (somewhat) in the statement of the theorem below by maintaining the names $P_{2}$ and $Q_{0}$ for a factorization through a quotient.

Theorem $4.6 \quad(1) \quad \varepsilon_{0 *}: L_{0}(\mathbb{Z}[t]) \stackrel{\cong}{\rightrightarrows} L_{0}(\mathbb{Z})$.

(2) $P_{2}^{R[t]}: \widehat{H}_{1}\left(\mathbb{Z}_{2} ; R[t]\right) /\left(V_{2}-1\right) \widehat{H}_{1}\left(\mathbb{Z}_{2} ; R[t]\right) \cong L_{2}(R[t])$, if $R=\mathbb{Z}$ or $\mathbb{Z}_{2}$.

(3) $P_{2}^{\mathbb{Z}\left[t^{-}\right]}: \widehat{H}_{1}\left(\mathbb{Z}_{2} ; \mathbb{Z}\left[t^{-}\right]\right) /\left\{p^{2}-p: p \in \widehat{H}_{1}\left(\mathbb{Z}_{2} ; \mathbb{Z}\left[t^{-}\right]\right)\right\} \cong L_{2}\left(\mathbb{Z}\left[t^{-}\right]\right)$.

(4) $Q_{0}^{\mathbb{Z}\left[t^{-}\right]}: t^{2} \widehat{H}_{1}\left(\mathbb{Z}_{2} ; \mathbb{Z}\left[t^{-}\right]\right) /\left\{p^{2}-p: p \in t^{2} \widehat{H}_{1}\left(\mathbb{Z}_{2} ; \mathbb{Z}\left[t^{-}\right]\right)\right\} \stackrel{\cong}{\rightrightarrows} N^{-} L_{2}\left(\mathbb{Z}\left[t^{-}\right]\right)$.

The isomorphism in (1) is of abelian groups, in (2) of $\mathcal{V}$-modules, and in (3) and (4) of $\mathcal{V}_{\text {od }}$-modules.

To prove this, we will need the following lemma, which is similar to 23. Proposition 41.3(v)] and [11, Proposition 2.11(ii)].

Lemma 4.7 Let $R$ be a principal ideal domain with involution. Any $\xi \in$ $\operatorname{ker}\left(\varepsilon_{0 *}: L_{2 n}\left(R\left[t^{ \pm}\right]\right) \rightarrow L_{2 n}(R)\right)$ can be represented by a $(-1)^{n}$-quadratic form $(M[t], \lambda, \mu)$ for which there is a free $R$-summand $L \subset M$, such that $L[t]=L[t]^{\perp}$ (a "symmetric Lagrangian").

Proof By Higman Linearization ([10, Lemma 3.6ab], also [21, Proposition 5.1.3]), extended to the case $t \mapsto-t$, one can represent $\xi$ by a form $(M[t], \lambda, \mu)$ $=\left(M[t], \lambda_{0}+t \lambda_{1}, \mu_{0}+t \mu_{1}\right)$. Here $M$ is a finitely generated free $R$-module; $M[t]=R[t] \otimes_{R} M$; and $\lambda_{0}(x, y), \lambda_{1}(x, y)$ are $R$-valued. A similar interpretation holds for $\mu_{0}+t \mu_{1}$. 
It follows that $\left(M, \lambda_{0}, \mu_{0}\right)$ is a $(-1)^{n}$-quadratic form and $\left(M, \lambda_{1}, \mu_{1}\right)$ is an $\varepsilon(-1)^{n}$-quadratic form where $t \mapsto \varepsilon t$. Since $\lambda_{0}$ and $\lambda_{0}+t \lambda_{1}$ are nonsingular, the $R$-map $\nu=\left(\operatorname{Ad} \lambda_{0}\right)^{-1} \circ \operatorname{Ad} \lambda_{1}: M \rightarrow M$ is nilpotent and satisfies:

$$
\lambda_{1}(x, y)=\lambda_{0}(\nu x, y)=\varepsilon \lambda_{0}(x, \nu y), \quad \text { for all } x, y \in M .
$$

Choose $k \geq 0$ so that $\nu^{k+1}=0$, but $\nu^{k} \neq 0$. First assume $\nu>0$. Let

$$
\overline{\nu^{k}(M)}=\left\{x \in M: a x \in \nu^{k}(M) \text { for some } a \in R-0\right\} \text {. }
$$

This is a summand of $M$. Pick a basis element $e_{1} \in \overline{\nu^{k} M}$. Then $\nu\left(e_{1}\right)=0$, and $\lambda_{0}\left(e_{1}, e_{1}\right)=0$.

Set $m=\left(M, \lambda_{0}\right), S_{1}=R e_{1}$. Then $S_{1}$ is a subLagrangian for $m$ and $\nu$ induces a nilpotent map $\nu_{S_{1}}: S_{1}^{\perp} / S_{1} \rightarrow S_{1}^{\perp} / S_{1}$. Repeat this step on the subLagrangian construction $m_{S_{1}}=\left(S_{1}^{\perp} / S_{1},\left(\lambda_{0}\right)_{S_{1}}\right)$, getting $e_{2}$, etc., until one obtains a basis $\left\{e_{1}, e_{2}, \ldots, e_{m}\right\}$ for a summand $S$ of $M$ satisfying:

$$
S \subset S^{\perp}, \quad \nu\left(S^{\perp}\right) \subset S .
$$

The Witt class of $m_{S}=\left(S^{\perp} / S,\left(\lambda_{0}\right)_{S}\right)$ is zero because $\xi \in \operatorname{ker}\left(\varepsilon_{0 *}\right)$. So by adding a hyperbolic form, if necessary, to the original $(M[t], \lambda, \mu)$, we may as well assume $m_{S}$ is hyperbolic. We can therefore find additional elements, $e_{m+1}, \ldots, e_{k} \in S^{\perp}$, whose images in $S^{\perp} / S$ form a basis for a Lagrangian of $m_{S}$. It follows that $L=<e_{1}, \ldots, e_{k}>$ is a summand of $M$ satisfying:

$$
L^{\perp}=L\left(\text { relative to } \lambda_{0}\right) ; \quad \nu(L) \subset L .
$$

Therefore $L[t]=L[t]^{\perp}$ (relative to $\left.\lambda\right)$.

Proof of Theorem 4.6 Let $R=\mathbb{Z}$ or $\mathbb{Z}_{2}$. Let $n=0$ or 1 . Let $\xi \in$ $N^{ \pm} L_{2 n}(R)=\operatorname{ker}\left(\varepsilon_{0 *}: L_{2 n}\left(R\left[t^{ \pm}\right] \rightarrow L_{2 n}(R)\right)\right.$. By Lemma 4.7 we may write $\xi=[M[t], \lambda, \mu]$, where $M$ is a free $R$-module containing a free summand $L$ for which $L[t]=L[t]^{\perp}$. Since $\lambda$ is an even form, any $R$-basis $\left\{e_{1}, \ldots, e_{k}\right\}$ for $L$ extends to an $R[t]$-symplectic basis $\left\{e_{1}, \ldots, e_{k}, f_{1}, \ldots, f_{k}\right\}$ for $(M[t], \lambda)$. So

$$
\xi=\sum_{i=1}^{k}\left[P_{p_{i}, q_{i}}\right] \text { where } p_{i}=\mu\left(e_{i}\right), q_{i}=\mu\left(f_{i}\right) .
$$

We use this expression for $\xi$ repeatedly below.

Our first step is to show that the maps $P_{2}^{R\left[t^{ \pm}\right]}$and $Q_{0}^{\mathbb{Z}\left[t^{-}\right]}$of Theorem 4.6] are surjective. We start with $P_{2}^{R\left[t^{ \pm}\right]}$. Note that $P_{2}^{R\left[t^{ \pm}\right]}[1]=\left[P_{1,1}\right]$ generates the image of $L_{2}(R)$, so we just need to show that every $\xi \in N^{ \pm} L_{2}(R)$ is in the 
image of $P_{2}$. By the first paragraph of this proof and parts (6) and (2) of Lemma 4.3 .

$$
\xi=\sum_{i=1}^{k}\left[P_{p_{i}, q_{i}}\right]=\sum_{i=1}^{k}\left[P_{p_{i} q_{i}, 1}\right]=P_{2}^{R\left[t^{ \pm}\right]}\left(\sum_{i=1}^{k} p_{i} q_{i}\right) .
$$

To see $Q_{0}^{\mathbb{Z}\left[t^{-}\right]}$is surjective, consider $\xi=\sum_{i=1}^{k}\left[P_{p_{i}, q_{i}}\right] \in N^{-} L_{0}(\mathbb{Z})$ as before. Note $p_{i}, q_{i} \in \widehat{H}_{0}\left(\mathbb{Z}_{2} ; \mathbb{Z}\left[t^{-}\right]\right)=t \mathbb{Z}_{2}\left[t^{2}\right]$. Therefore, write $p_{i} \equiv r_{i} t \bar{r}_{i}\left(\bmod 2 \mathbb{Z}\left[t^{-}\right]\right)$ and $q_{i} \equiv s_{i} t \bar{s}_{i}\left(\bmod 2 \mathbb{Z}\left[t^{-}\right]\right)$where $r_{i}, s_{i} \in \mathbb{Z}\left[t^{-}\right]$. Then, with the help of Lemma $4.3(5)$, one sees

$$
\xi=\sum_{i=1}^{k}\left[P_{p_{i}, q_{i}}\right]=\sum_{i=1}^{k}\left[P_{r_{i} t \bar{r}_{i}, s_{i} t \bar{s}_{i}}\right]=\sum_{i=1}^{k}\left[P_{\bar{s}_{i} r_{i} t \bar{r}_{i} s_{i}, t}\right]=Q_{0}^{\mathbb{Z}\left[t^{-}\right]}\left(\sum_{i=1}^{k} \bar{s}_{i} r_{i} t^{2} \bar{r}_{i} s_{i}\right) .
$$

Now we need to prove injectivity of the four maps. We start with $\varepsilon_{0 *}$. By the first paragraph of the proof, every $\xi \in \operatorname{ker}\left(\varepsilon_{0 *}: L_{0}\left(\mathbb{Z}[t] \rightarrow L_{0}(\mathbb{Z})\right)\right.$ can be represented by a quadratic form $(M[t], \lambda, \mu)$ where $M[t]$ has a free summand $L[t]$ so that for all $x \in L[t]$, one has $\lambda(x, x)=0$. But $2 \mu(x)=\lambda(x, x) \in \mathbb{Z}[t]$, so $\left.\mu\right|_{L[t]}=0$. Thus $L[t]$ is a Lagrangian and so $\xi=0$. Hence $\varepsilon_{0 *}$ is injective.

For a field $F$ of characteristic 2, there is a well-defined epimorphism called the Arf invariant (see [1, 24])

$$
\text { Arf }: L_{2 n}(F) \rightarrow F /\left\{x^{2}+x: x \in F\right\}, \quad[M, \lambda, \mu] \mapsto \sum_{i=1}^{k} \mu\left(e_{i}\right) \mu\left(f_{i}\right),
$$

where $\left\{e_{1}, \ldots e_{k}, f_{1}, \ldots f_{k}\right\}$ is a symplectic basis for $(M, \lambda)$. Applying this to the function field $\mathbb{Z}_{2}(t)$ and then restricting to the subring $\mathbb{Z}_{2}[t]$, one obtains an epimorphism

$$
\text { arf : } L_{2 n}\left(\mathbb{Z}_{2}[t]\right) \rightarrow \mathbb{Z}_{2}[t] /\left\{x^{2}+x: x \in \mathbb{Z}_{2}[t]\right\} .
$$

(Note, there is a subtle point here - one needs that $\mathbb{Z}_{2}[t] /\left\{x^{2}+x: x \in \mathbb{Z}_{2}[t]\right\} \rightarrow$ $\mathbb{Z}_{2}(t) /\left\{x^{2}+x: x \in \mathbb{Z}_{2}(t)\right\}$ is injective. This can be shown using the fact that $\mathbb{Z}_{2}[t]$ is a PID and hence integrally closed.)

We now return to the proof of injectivity of $P_{2}^{R[t]}$ where $R=\mathbb{Z}$ or $\mathbb{Z}_{2}$. Let $r: L_{2}(R[t]) \rightarrow L_{2}\left(\mathbb{Z}_{2}[t]\right)$ be mod 2 reduction or the identity map. Then $P_{2}^{R[t]}$ is injective since arf $\circ r \circ P_{2}^{R[t]}$ is the identity map.

To show that $P_{2}^{\mathbb{Z}\left[t^{-}\right]}$is injective we consider $\bmod 2$ reduction $r: L_{2}\left(\mathbb{Z}\left[t^{-}\right]\right) \rightarrow$ $L_{2}\left(\mathbb{Z}_{2}[t]\right)$. We will show

$$
\operatorname{arf} \circ r \circ P_{2}^{\mathbb{Z}\left[t^{-}\right]}: \mathbb{Z}_{2}\left[t^{2}\right] /\left\{x^{2}-x: x \in \mathbb{Z}_{2}\left[t^{2}\right]\right\} \rightarrow \mathbb{Z}_{2}\left[t^{2}\right] /\left\{x^{2}-x: x \in \mathbb{Z}_{2}[t]\right\}
$$


is injective. Suppose $\left(\operatorname{arf} \circ r \circ P_{2}^{\mathbb{Z}\left[t^{-}\right]}\right)[p]=[p]=0$ for some $p \in \mathbb{Z}_{2}\left[t^{2}\right]$. This last equality means $p=x^{2}-x$ for some $x \in \mathbb{Z}_{2}\left[t^{2}\right]$. Since $x^{2}$ and $x^{2}-x$ are both in $\mathbb{Z}_{2}\left[t^{2}\right]$, we see $x \in \mathbb{Z}_{2}\left[t^{2}\right]$ and thus $[p]=0 \in \mathbb{Z}_{2}\left[t^{2}\right] /\left\{x^{2}-x: x \in \mathbb{Z}_{2}\left[t^{2}\right]\right\}$. Hence $P_{2}^{\mathbb{Z}\left[t^{-}\right]}$is injective.

To show that $Q_{0}^{\mathbb{Z}\left[t^{-}\right]}$is injective we consider $\bmod 2$ reduction $r: L_{0}\left(\mathbb{Z}\left[t^{-}\right]\right) \rightarrow$ $L_{0}\left(\mathbb{Z}_{2}[t]\right)$. We will show

$$
\operatorname{arf} \circ r \circ Q_{0}^{\mathbb{Z}\left[t^{-}\right]}: t^{2} \mathbb{Z}_{2}\left[t^{2}\right] /\left\{x^{2}-x: x \in t^{2} \mathbb{Z}_{2}\left[t^{2}\right]\right\} \rightarrow \mathbb{Z}_{2}\left[t^{2}\right] /\left\{x^{2}-x: x \in \mathbb{Z}_{2}[t]\right\}
$$

is injective. Suppose $\left(\operatorname{arf} \circ r \circ Q_{0}^{\mathbb{Z}\left[t^{-}\right]}\right)\left[t^{2} p\right]=\left[t^{2} p\right]=0$ for some $p \in \mathbb{Z}_{2}\left[t^{2}\right]$. This last equality means $t^{2} p=x^{2}-x$ for some $x \in \mathbb{Z}_{2}\left[t^{2}\right]$. Since $x^{2}$ and $x^{2}-x$ are both in $\mathbb{Z}_{2}\left[t^{2}\right]$, we see $x \in \mathbb{Z}_{2}\left[t^{2}\right]$. Set $x=c+y$ where $c \in \mathbb{Z}_{2}$ and $y \in t^{2} \mathbb{Z}_{2}\left[t^{2}\right]$. Clearly $y^{2}-y=\left(x^{2}-x\right)+\left(c^{2}-c\right)=x^{2}-x=t^{2} p$. Thus $\left[t^{2} p\right]=0$ in the domain. Hence $Q_{0}^{\mathbb{Z}\left[t^{-}\right]}$is injective.

We can now calculate $\mathcal{L}^{\mathrm{ev}}\left(\mathbb{Z}\left[t^{ \pm}\right], 2\right)$.

Theorem 4.8 (1) There is an isomorphism of $\mathcal{V}$-modules,

$$
\begin{aligned}
& \mathcal{V} /\left\langle 2, V_{2}-1\right\rangle \cong \frac{t \mathbb{Z}_{2}[t]}{\left(V_{2}-1\right) t \mathbb{Z}_{2}[t]} \stackrel{\mathcal{P}}{\cong} \widetilde{\mathcal{L}}^{\mathrm{ev}}(\mathbb{Z}[t], 2) ; \\
& \mathcal{P}[p]=\left[\mathbb{Z}_{2}[t]^{2},\left(\begin{array}{cc}
0 & 1 / 2 \\
1 / 2 & 0
\end{array}\right)\left(\begin{array}{l}
p \\
1
\end{array}\right)\right] . \\
& \widetilde{\mathcal{L}}^{\mathrm{ev}}\left(\mathbb{Z}\left[t^{-}\right], 2\right)=0 .
\end{aligned}
$$

Proof (1) By Proposition 4.2, $\alpha_{*}: \widetilde{L}_{0}\left(\mathbb{Z}_{2}[t]\right) \rightarrow \widetilde{\mathcal{L}}^{\mathrm{ev}}(\mathbb{Z}[t], 2)$ is an isomorphism. Then $\left(\alpha_{*}\right)^{-1} \circ \mathcal{P}$ is the isomorphism of Theorem 4.6(2).

(2) Let $m=(M, b, q)$ be a quadratic linking form of exponent 2 with even type over $\mathbb{Z}\left[t^{-}\right]$. Then $M$ admits a symplectic basis over $\mathbb{Z}_{2}[t]$. This means $m$ is an orthogonal sum of terms of the form $\mathcal{P}_{p, g}$, where $p, g \in \mathbb{Z}\left[t^{2}\right]$. By parts (2) and (4) of Lemma 4.3, $[m]$ is a sum of terms of the form $\left[\mathcal{P}_{t^{4 n+2}, 1}\right]$.

But $\left[\mathcal{P}_{t^{4 n+2}, 1}\right]=0$ in $\widetilde{\mathcal{L}}\left(\mathbb{Z}\left[t^{-}\right], 2\right)$, because $\left\langle e+t^{2 n+1} f\right\rangle$ is a Lagrangian for $\mathcal{P}_{t^{4 n+2}, 1}$. To see this, we compute:

$$
q\left(e_{1}+t^{2 n+1} e_{2}\right)=t^{4 n+2}+t^{4 n+2}+\{1+*\}\left(t^{2 n+1} / 2\right)=\{1+*\}\left(t^{2 n+1} / 2\right)=0 .
$$

This proves $\widetilde{\mathcal{L}}^{\mathrm{ev}}\left(\mathbb{Z}\left[t^{-}\right], 2\right)=0$. 


\section{Characteristic elements and the proof of Theorem 1.5}

In this section we write $R$ for $\mathbb{Z}[t], \mathbb{Z}\left[t^{-}\right]$, or $\mathbb{Z}$. For any $R$-module $M$ and any $k \in \mathbb{N}$ we write

$$
{ }_{k} M=\{x \in M: k x=0\} .
$$

Let $N$ be a submodule of a length one $R$-module $M$ of exponent $2^{n}$, for some $n$. The closure of $N$ in $M$ is:

$$
\bar{N}=\{x \in M: p x \in N \text { for some } p \in R \backslash 2 R\} .
$$

By Corollary A.5 of the Appendix, $\bar{N}$ is the unique smallest colength one submodule of $M$ which contains $N$. We will use this concept below.

The goal of this section is to prove:

Theorem 5.1 Let $R=\mathbb{Z}\left[t^{ \pm}\right]$or $\mathbb{Z}$. There is a short exact sequence:

$$
0 \rightarrow \mathcal{L}(R, 2) \rightarrow \mathcal{L}(R,\langle 2\rangle) \stackrel{Q}{\longrightarrow} \mathbb{Z}_{2} \rightarrow 0
$$

Moreover, $\mathcal{L}\left(R, 2^{n}\right) \rightarrow \mathcal{L}\left(R, 2^{n+1}\right)$ is an isomorphism if $n \geq 2$.

We will then prove Theorem 1.5] as a corollary of Theorem 5.1]

Construction of Characteristic Elements $\mathbf{v}_{\mathbf{o}}, \mathbf{v}_{\mathbf{1}}$ Fix an integer $n \geq 1$ and a quadratic linking form $m=(M, b, q)$ for which $2^{n} M=0$. We construct elements $v_{0}^{(n)}(m), v_{1}^{(n)}(m) \in{ }_{2} M$. They depend on $M, b$, and $n$.

Recall that $\widehat{H}_{1}\left(\mathbb{Z}_{2} ; R\right)$ is an $R / 2 R$-module via $[a] \circ[x]=[a x \bar{a}]$. Consider the $R$-linear map

$$
\phi: M \rightarrow \widehat{H}_{1}\left(\mathbb{Z}_{2} ; R\right), \quad \phi(x)=\{1+*\} 2^{n-1} b(x, x)=2^{n} q(x) \in \widehat{H}_{1}\left(\mathbb{Z}_{2} ; R\right) .
$$

Here $\{1+*\}: \frac{1}{2} R / R \rightarrow \widehat{H}_{1}\left(\mathbb{Z}_{2} ; R\right)$ is given by $[x] \mapsto[x+\bar{x}] \in \widehat{H}_{1}\left(\mathbb{Z}_{2} ; R\right)$. It is bijective if $R=\mathbb{Z}[t]$ or $\mathbb{Z}$.

The map $\phi$ measures the failure of $m$ to be of even type.

As an $R / 2 R$-module, $\widehat{H}_{1}\left(\mathbb{Z}_{2} ; R\right)$ is free on the basis $\{[1],[t]\}$ if $R=\mathbb{Z}[t]$ and on the basis $\{[1]\}$ if $R=\mathbb{Z}\left[t^{-}\right]$or $\mathbb{Z}$. Therefore, if $R=\mathbb{Z}[t]$, there are $R$-maps $\phi_{0}, \phi_{1}: M \rightarrow R / 2 R$, uniquely specified by the rule

$$
\phi(x)=\phi_{0}(x) \circ[1]+\phi_{1}(x) \circ[t] .
$$


If $R=\mathbb{Z}\left[t^{-}\right]$or $\mathbb{Z}$, we get a single map $\phi_{0}: M \rightarrow R / 2 R$, uniquely specified by

$$
\phi(x)=\phi_{0}(x) \circ[1] .
$$

We define $\phi_{1}$ to be 0 in these cases.

The map Ad $b: M \rightarrow M^{\wedge}$ restricts to an isomorphism

$$
{ }_{2} M \stackrel{\cong}{\rightrightarrows}{ }_{2}\left(M^{\wedge}\right)=\operatorname{Hom}_{R}\left(M, \frac{1}{2} R / R\right) .
$$

We will change the target using the isomorphism $\{2\}: \frac{1}{2} R / R \cong R / 2 R$ sending $x+R$ to $2 x+2 R$.

Therefore there are elements $v_{i}=v_{i}^{(n)}(m) \in{ }_{2} M$, defined by

$$
v_{i}=(\operatorname{Ad} b)^{-1}\left(\{2\}^{-1} \circ \phi_{i}\right) \text {. }
$$

In other words

$$
\{2\} b\left(v_{i}, x\right)=\phi_{i}(x) \text { for all } x \in M \text {. }
$$

If $R=\mathbb{Z}\left[t^{-}\right]$or $\mathbb{Z}$, then $v_{1}=0$.

We conclude that $v_{0}, v_{1} \in{ }_{2} M$ are characterized by the fact that for all $x \in M$ :

$$
\begin{aligned}
& \{2\} 2^{n-1} b(x, x)=\left(\{2\} b\left(v_{0}, x\right)\right)^{2}+\left(\{2\} b\left(v_{1}, x\right)\right)^{2} t \in \mathbb{Z}_{2}[t], \text { if } R=\mathbb{Z}[t], \\
& \{2\} 2^{n-1} b(x, x)=\left(\{2\} b\left(v_{0}, x\right)\right)^{2} \in \mathbb{Z}_{2}\left[t^{2}\right], \text { if } R=\mathbb{Z}\left[t^{-}\right], \\
& \{2\} 2^{n-1} b(x, x)=\left(\{2\} b\left(v_{0}, x\right)\right)^{2}=\{2\} b\left(v_{0}, x\right) \in \mathbb{Z}_{2}, \text { if } R=\mathbb{Z} .
\end{aligned}
$$

Proposition 5.2 The construction above satisfies the following properties:

(1) If $m=m^{\prime} \oplus m^{\prime \prime}$ (orthogonal direct sum), then

$$
v_{i}^{(n)}\left(m^{\prime} \oplus m^{\prime \prime}\right)=v_{i}^{(n)}\left(m^{\prime}\right) \oplus v_{i}^{(n)}\left(m^{\prime \prime}\right) .
$$

(2) Suppose $L \subset M$ and $2^{n-1} b(x, x)=0$, for all $x \in L$. Then $v_{i}^{(n)}(m) \in L^{\perp}$. So if $L$ is a Lagrangian for $m$, then $v_{i}^{(n)}(m) \in L$, and $q\left(v_{i}^{(n)}(m)\right)=0$ for each $i$.

$$
v_{i}^{(n)}(m) \in \overline{2^{n-1} M} \text {, for } i=0,1 .
$$

Proof (1) This is obvious from the definitions.

(2) This is clear from (4), (5), and (6), because $\phi(L)=0$.

(3) In general, $\left(2^{k} M\right)^{\perp}={ }_{2^{k}} M$. This implies $\left({ }_{2^{k}} M\right)^{\perp}=\left(2^{k} M\right)^{\perp \perp}=\overline{\left(2^{k} M\right)}$, by Proposition A.6 in the Appendix. So we must only show that $v_{i} \in\left({ }_{2^{n-1}} M\right)^{\perp}$, for $i=0,1$. But this is clear from (2) above, if we set $L={ }_{2^{n-1}} M$. 
There is one crucial case in which we can slightly strengthen Proposition $5.2(3)$, which we describe now. A quadratic linking form $(M, b, q)$ is irreducible if for all $x \neq 0$ in $M$, we have $q(x) \neq 0$.

Lemma 5.3 Let $m=(M, b, q)$ be an irreducible quadratic linking form over $R$, with $4 M=0$. Then $v_{i}^{(2)}(m) \in 2 M$, for each $i$.

Proof We assume $R=\mathbb{Z}\left[t^{ \pm}\right]$; in the $R=\mathbb{Z}$ case there is nothing to prove by Proposition $[5.2(3)$. For any $p \in \mathbb{Z}[t] \backslash 2 \mathbb{Z}[t]$, let $M(p)$ denote the $\mathbb{Z}[t]$-module generated by two elements $\phi, \tau$, subject only to the two relations:

$$
2 \tau=0, \quad 2 \phi=p \tau .
$$

Note that $M(p)$ can also be viewed as the submodule $\langle[p],[2]\rangle$ of $\mathbb{Z}_{4}[t]$. Hence by Corollary A.4 $M(p)$ has length one.

Its dual, $M(p)^{\wedge}=\operatorname{Hom}_{R}(M, \mathbb{Q} R / R)$, is generated by two homomorphisms $\Phi, T$ defined by:

$$
\Phi(\phi)=\frac{p}{4}, \quad \Phi(\tau)=\frac{1}{2}=T(\phi), \quad T(\tau)=0 .
$$

Note $M(p) \cong M(p)^{\wedge}$.

Since $4 M=0$, multiplication by 2 gives a monomorphism $\times 2: M /{ }_{2} M \rightarrow{ }_{2} M$ of finitely generated torsion-free modules over the principal ideal domain $\mathbb{Z}_{2}[t]$. By the structure theorem for such modules, we can find bases $\left\{\tau_{1}, \tau_{2}, \ldots, \tau_{l}\right\}$ for ${ }_{2} M$, and $\left\{\left[\phi_{1}\right],\left[\phi_{2}\right], \ldots,\left[\phi_{k}\right]\right\}$ for $M /{ }_{2} M$ over $\mathbb{Z}_{2}[t]$, (where $\phi_{i} \in M, i=$ $1, \ldots, k)$, so that $2 \phi_{i}=p_{i} \tau_{i}$ for some $p_{i} \in \mathbb{Z}[t] \backslash 2 \mathbb{Z}[t]$, whose class $\left[p_{i}\right] \in \mathbb{Z}_{2}[t]$ divides $\left[p_{i+1}\right]$, for $0<i<k$. Note that $0 \leq k \leq l$.

It follows that $M=M\left(p_{1}\right) \oplus M\left(p_{2}\right) \oplus \ldots M\left(p_{k}\right) \oplus N$, where $N \cong \mathbb{Z}_{2}[t]^{l-k}$ is the submodule with basis $\left\{\tau_{k+1}, \ldots, \tau_{l}\right\}$. Therefore, $M^{\wedge}$ is spanned by $\Phi_{1}, \ldots, \Phi_{k}, T_{1}, \ldots, T_{l}$, and $N^{\wedge}$ is spanned by $T_{k+1}, \ldots, T_{l}$.

Now $\operatorname{rank}_{\mathbb{Z}_{2}[t]}\left(\widehat{H}_{1}\left(\mathbb{Z}_{2} ; R\right)\right)=1$ or 2 . Since $m$ is irreducible,

$$
4 q: M /{ }_{2} M \rightarrow \widehat{H}_{1}\left(\mathbb{Z}_{2} ; R\right)
$$

is an $\mathbb{Z}_{2}[t]$-linear monomorphism. So $k \leq 2$ if $R=\mathbb{Z}[t] ; k \leq 1$ if $R=\mathbb{Z}\left[t^{-}\right]$. The rest of the proof breaks into cases depending on the value of $\mathrm{k}$.

Case One Assume $k=0$. By Proposition $\left[5.2(3), v_{i}^{(2)}(m) \in \overline{2 M}\right.$, but $\overline{2 M}=$ $2 M$ since $2 M=0$.

Case Two Assume $k=1$. We have $M=M\left(p_{1}\right) \oplus N$. Because $b$ is nonsingular, $(\operatorname{Ad} b)\left(\phi_{1}\right) \equiv \Phi_{1}\left(\bmod { }_{2}\left(M^{\wedge}\right)\right)$, noting that $M /{ }_{2} M$ and $M^{\wedge} /{ }_{2}\left(M^{\wedge}\right)$ are 
both free and rank 1 over $\mathbb{Z}_{2}[t]$ with bases $\phi_{1}$ and $\Phi_{1}$. Therefore, $2 b\left(\phi_{1}, \phi_{1}\right)=$ $\frac{p_{1}}{2}$. We have:

$$
p_{1}=\{2\} 2 b\left(\phi_{1}, \phi_{1}\right)=4 q\left(\phi_{1}\right)=q\left(p_{1} \tau_{1}\right)=\left(p_{1}\right)^{2} q\left(\tau_{1}\right) \in \mathbb{Z}_{2}[t] .
$$

So $p_{1} q\left(\tau_{1}\right)=1$ in $\mathbb{Z}_{2}[t]$. In particular, $\left[p_{1}\right]=1 \in \mathbb{Z}_{2}[t]$, and so $2 M=\overline{2 M}$ since $M=\mathbb{Z}_{4}[t] \oplus \mathbb{Z}_{2}[t]^{j}$. So again Proposition $[5.2(3)$ proves the result.

Case Three Assume $k=2$. Hence $R=\mathbb{Z}[t]$ and $M=M\left(p_{1}\right) \oplus M\left(p_{2}\right) \oplus N$.

Claim $\left[p_{1}\right]=\left[p_{2}\right] \in \mathbb{Z}_{2}[t]$.

Proof of Claim Write $\left[p_{2}\right]=\left[p_{1} r\right]$ where $r \in \mathbb{Z}[t]$. Then let $A: \overline{2 M} \cong \overline{2 M^{\wedge}}$ be the restriction of the isomorphism Ad $b: M \rightarrow M^{\wedge}$. Let $\left(\begin{array}{ll}\alpha & \beta \\ \gamma & \delta\end{array}\right) \in G L_{2}\left(\mathbb{Z}_{2}[t]\right)$ be the matrix of $A$ relative to the bases $\left\{\tau_{1}, \tau_{2}\right\}$ and $\left\{T_{1}, T_{2}\right\}$ for $\overline{2 M}$ and $\overline{2 M^{\wedge}}$ respectively. We then have

$$
\begin{aligned}
& \frac{p_{1}}{2} \gamma=p_{1} \gamma T_{2}\left(\phi_{2}\right)=p_{1} A\left(\tau_{1}\right)\left(\phi_{2}\right)=p_{1} b\left(\tau_{1}, \phi_{2}\right)=p_{1} b\left(\phi_{2}, \tau_{1}\right)= \\
& b\left(2 \phi_{2}, \phi_{1}\right)=p_{2} A\left(\tau_{2}\right)\left(\phi_{1}\right)=p_{1} r \beta T_{1}\left(\phi_{1}\right)=\frac{p_{1}}{2} r \beta \in \frac{1}{2} \mathbb{Z}[t] / \mathbb{Z}[t] .
\end{aligned}
$$

Therefore $\gamma=r \beta$. The matrix $\left(\begin{array}{cc}\alpha & \beta \\ r \beta & \delta\end{array}\right)$ is therefore nonsingular, $\alpha \delta=1+r \beta^{2}$, and consequently $\delta$ is relatively prime to $[r]$.

On the other hand,

$$
p_{1} r \delta=p_{2} \delta=p_{2}\{2\} \frac{\delta}{2}=\{2\} b\left(p_{2} \tau_{2}, \phi_{2}\right)=4 q\left(\phi_{2}\right)=q\left(p_{2} \tau_{2}\right)=p_{1}^{2} r^{2} q\left(\tau_{2}\right) \in \mathbb{Z}_{2}[t] .
$$

Therefore $\delta=p_{1} r q\left(\tau_{2}\right)$, and so $[r]$ obviously divides $\delta$. This implies $[r]=1 \in$ $\mathbb{Z}_{2}[t]$, proving the claim.

Returning to the proof of Case Three, we write $M=M(p) \oplus M(p) \oplus N$. Note that $4 q\left(\phi_{i}\right)=p^{2} q\left(\tau_{i}\right), i=1,2$. Therefore $4 q(M) \subset p \circ \widehat{H}_{1}\left(\mathbb{Z}_{2} ; \mathbb{Z}[t]\right)$, and so $\{2\} b\left(v_{i}, M\right) \subset p \mathbb{Z}_{2}[t]$, which tells us $v_{i} \in p M$. But, by construction of $M$ we see $p M \cap \overline{2 M}=2 M$, so by Proposition [5.2(3) again, $v_{i} \in 2 M$, for $i=0,1$. This completes the proof.

Remark 5.4 A study of the above proof shows that $M(p)$ has the following two cool properties. Each length one $\mathbb{Z}[t]$-module of exponent four is isomorphic to a direct $\operatorname{sum} M\left(p_{1}\right) \oplus \ldots M\left(p_{k}\right) \oplus \mathbb{Z}_{2}[t]^{j}$. If $[p] \neq 0,1$, then $M(p)$ admits a nonsingular bilinear linking form, but no quadratic linking form. 
Our proof of Theorem 5.1 is largely accomplished by the following definition and the next two lemmas.

Definition $5.5\left(\right.$ of $\left.Q_{0}^{(n)}, Q_{1}^{(n)}: \mathcal{L}\left(R, 2^{n}\right) \rightarrow \widehat{H}_{1}\left(\mathbb{Z}_{2} ; R\right)\right)$

Let $m=(M, b, q)$ be a quadratic linking form over $R$ with $2^{n} M=0$. By Proposition $\left[5.2(3)\right.$, the characteristic elements satisfy $v_{i}=v_{i}^{(n)}(m) \in \overline{2^{n-1} M}$. Therefore if $n \neq 1, b\left(v_{i}, v_{i}\right)=0$ for each $i$, so that $q\left(v_{i}\right) \in \widehat{H}_{1}\left(\mathbb{Z}_{2} ; R\right)$. (Recall $\widehat{H}_{1}\left(\mathbb{Z}_{2} ; R\right)$ is $\mathbb{Z}_{2}[t]$ or $\mathbb{Z}_{2}\left[t^{2}\right]$ or $\left.\mathbb{Z}_{2}.\right)$ We set

$$
\begin{aligned}
& Q_{i}^{(n)}(m)=q\left(v_{i}\right) \in \widehat{H}_{1}\left(\mathbb{Z}_{2} ; R\right), \text { if } n>1, \\
& Q_{i}^{(1)}(m)=q\left(v_{i}\right) \in \frac{1}{2}\{x \in R: x=\bar{x}\} /(1+*) R, \text { if } n=1 .
\end{aligned}
$$

Then $Q_{i}^{(n)}$ is additive, and $Q_{i}^{(n)}(m)=0$ if $m$ admits a Lagrangian, by Proposition $\left[5.2(2)\right.$. So each $Q_{i}^{(n)}$ is a well-defined homomorphism on $\mathcal{L}\left(R, 2^{n}\right)$.

Obviously, if $R=\mathbb{Z}$ or $\mathbb{Z}\left[t^{-}\right], Q_{1}^{(n)}=0$ for all $n$. We define

$$
\mathcal{L}^{\varepsilon}\left(R, 2^{n}\right)=\operatorname{ker} Q_{0}^{(n)} \cap \operatorname{ker} Q_{1}^{(n)} \subset \mathcal{L}\left(R, 2^{n}\right) .
$$

If $m$ is a quadratic linking form of exponent $2^{n}$ with even type, $v_{0}^{(n)}(m)=$ $v_{1}^{(n)}(m)=0$. Hence

$$
\iota_{n}\left(\mathcal{L}^{\mathrm{ev}}\left(R, 2^{n}\right)\right) \subset \mathcal{L}^{\varepsilon}\left(R, 2^{n}\right)
$$

Lemma 5.6 (1) For all $n \geq 3, \quad \mathcal{L}^{\varepsilon}\left(R, 2^{n}\right)=\mathcal{L}\left(R, 2^{n}\right)$.

(2) Furthermore $Q_{1}^{(2)}=0$, and there is a short exact sequence:

$$
0 \rightarrow \mathcal{L}^{\varepsilon}(R, 4) \rightarrow \mathcal{L}(R, 4) \stackrel{Q_{0}^{(2)}}{\longrightarrow} \mathbb{Z}_{2} \rightarrow 0 .
$$

(3) Finally, for each $i, Q_{i}^{(1)}(\widetilde{\mathcal{L}}(R, 2)) \subset \widehat{H}_{1}\left(\mathbb{Z}_{2} ; R\right)$.

Proof (1) First suppose $n \geq 3$, and $[m]=[M, b, q] \in \mathcal{L}\left(R, 2^{n}\right)$. We show for each $i$, that $q\left(v_{i}^{(n)}(m)\right)=0$. This will prove $\mathcal{L}^{\varepsilon}\left(R, 2^{n}\right)=\mathcal{L}\left(R, 2^{n}\right)$.

By Proposition $\left[5.2(3), v_{i}^{(n)}(m) \in \overline{2^{n-1} M}\right.$. Therefore $p v_{i}^{(n)}(m) \in 2^{n-1} M$, for some $p \in R$ such that $[p] \neq 0$ in $R / 2 R$. Therefore $p^{2} q\left(v_{i}^{(n)}(m)\right) \in q\left(2^{n-1} M\right)=$ $2^{2 n-2} q(M)=0$, because $2 n-2 \geq n+1$. This implies $q\left(v_{i}^{(n)}(m)\right)=0$.

(2) Next we prove $Q_{1}^{(2)}=0$ and $\operatorname{im}\left(Q_{0}^{(2)}\right) \subset \mathbb{Z}_{2}$. 
Let $m=(M, b, q)$ be a quadratic linking form with $4 M=0$. Let $v_{i}=v_{i}^{(2)}(m)$. We must show that $q\left(v_{1}\right)=0$, and $q\left(v_{0}\right)=0$ or 1 . If we choose, we may replace $m$ by $m_{S}=\left(S^{\perp} / S, b_{S}, q_{S}\right)$, where $S$ is any subLagrangian of $m$. Moreover, $S=\overline{\langle x\rangle}$ is a subLagrangian, for any $x \in M$ for which $q(x)=0$, by Corollary A.5 of the Appendix. This means we may as well assume that $m$ is irreducible. Therefore, by Lemma [5.3 we may write $v_{0}=2 y_{0}, v_{1}=2 y_{1}$, for some $y_{0}, y_{1}$. Define $b_{i j}=\{2\} b\left(v_{i}, y_{j}\right)=b_{j i} \in R / 2 R$. Note

$$
b_{i i}=\{2\} 2 b\left(y_{i}, y_{i}\right)=4 q\left(y_{i}\right)=q\left(v_{i}\right) .
$$

From (41) we see

$$
b_{i i}=\{2\} 2 b\left(y_{i}, y_{i}\right)=b_{0 i}^{2}+b_{1 i}^{2} t .
$$

(When $R=\mathbb{Z}\left[t^{-}\right]$or $\mathbb{Z}$, these equations simplify to: $b_{00}=b_{00}^{2}$, which immediately gives $Q_{0}^{(2)}(m)=0$ or $\left.1, Q_{1}^{(2)}=0\right)$.

But, in the ring $R / 2 R=\mathbb{Z}_{2}[t]$, the equations

$$
\begin{aligned}
& b_{00}=b_{00}^{2}+b_{10}^{2} t \\
& b_{11}=b_{10}^{2}+b_{11}^{2} t
\end{aligned}
$$

have only two solutions: $0=b_{11}=b_{10} ; \quad b_{00}=0$ or 1 . For no polynomial of the form $p^{2}-p \in \mathbb{Z}_{2}[t]$ is an odd degree polynomial if $\operatorname{deg}(p)>0$. So $Q_{0}^{(2)}(m) \in$ $\{0,1\}, Q_{1}^{(2)}(m)=0$, and $\mathcal{L}^{\varepsilon}(R, 4)=\operatorname{ker}\left(Q_{0}^{(2)}\right) \cap \operatorname{ker}\left(Q_{1}^{(2)}\right)=\operatorname{ker}\left(Q_{0}^{(2)}\right)$.

To finish the proof of $(2)$, we note that $Q_{0}^{(2)}([m])=1$ when

$$
M=\mathbb{Z}_{4}\left[t^{ \pm}\right], \quad b(x, y)=\frac{x \bar{y}}{4}, \quad q(x)=\frac{x \bar{x}}{4} \quad \text { for all } x, y \in M .
$$

In this case $v_{0}=2$. Consequently, $Q_{0}^{(2)}(m)=q(2)=1$. This proves (2).

(3) This is similar to the argument for (2). Let $[m] \in \mathcal{L}(R, 2)$, and assume $\varepsilon_{0 *}([m])=0$. To show $Q_{i}^{1}(m) \in \widehat{H}_{1}\left(\mathbb{Z}_{2} ; R\right)$, we only have to show $2 q\left(v_{i}^{(1)}(m)\right)=$ 0 for each $i$. Set $v_{i}=v_{i}^{(1)}(m)$. This time set $a_{i j}=\{1+*\} b\left(v_{i}, v_{j}\right)$, so that $2 q\left(v_{i}\right)=a_{i i}$. Again from (44), (15), (6) we get

$$
\begin{aligned}
& a_{00}=a_{00}^{2}+a_{10}^{2} t \\
& a_{11}=a_{10}^{2}+a_{11}^{2} t .
\end{aligned}
$$

This yields again that $2 q\left(v_{1}\right)=a_{11}=0,2 q\left(v_{0}\right)=a_{00}=0$ or 1 , and $a_{10}=$ $b\left(v_{1}, v_{2}\right)=0$. But if $2 q\left(v_{0}\right)$ were equal to 1 , we would recall $\varepsilon_{0 *}([m])=0$ in $\mathcal{L}(\mathbb{Z}, 2)$, and conclude $1=\varepsilon_{0}(1)=\varepsilon_{0}\left(Q_{0}^{(1)}([m])=Q_{0}^{(1)}\left(\varepsilon_{0 *}[m]\right)=Q_{0}^{(1)}(0)\right.$, which is impossible. This proves $Q_{i}^{(1)}(\widetilde{\mathcal{L}}(R, 2)) \subset \widehat{H}_{1}\left(\mathbb{Z}_{2} ; R\right)$. 
Lemma 5.7 (1) For $n \geq 1, \mathcal{L}^{\mathrm{ev}}\left(R, 2^{n}\right) \stackrel{\iota_{n}}{\longrightarrow} \mathcal{L}^{\varepsilon}\left(R, 2^{n}\right)$ is an isomorphism.

(2) For $n \geq 2, \quad \mathcal{L}\left(R, 2^{n-1}\right) \stackrel{j_{n-1}}{\longrightarrow} \mathcal{L}^{\mathrm{ev}}\left(R, 2^{n}\right)$ is an isomorphism.

Proof Throughout this proof we use without comment Corollary A.5 which states that a submodule $N$ of a length one module $M$ has colength one if and only if $N=\bar{N}$. For example, a subLagrangian $S$ satisfies $S=\bar{S}$.

(1) We construct an inverse $G_{n}: \mathcal{L}^{\varepsilon}\left(R, 2^{n}\right) \rightarrow \mathcal{L}^{\mathrm{ev}}\left(R, 2^{n}\right)$ to $\iota_{n}$ for each $n \geq 1$. Let $[m]=[M, b, q] \in \mathcal{L}^{\varepsilon}\left(R, 2^{n}\right)$. Set $v_{i}=v_{i}^{(n)}(m)$. By definition of $\mathcal{L}^{\varepsilon}\left(R, 2^{n}\right)$ and by Proposition [5.2(3),

$$
T(m)=\overline{\left\langle v_{0}, v_{1}\right\rangle}
$$

is a subLagrangian. (If $n=1$ and $R=\mathbb{Z}[t]$, we must also use the conclusion $b\left(v_{1}, v_{0}\right)=0$ from the proof of Lemma [5.6). By the definition of $v_{i}$, we see $2^{n} q(x)=0$ for all $x \in T(m)^{\perp}$, and so $m_{T(m)}$ is of exponent $2^{n}$ with even type. We define

$$
g(m)=\left[m_{T(m)}\right] \in \mathcal{L}^{\mathrm{ev}}\left(R, 2^{n}\right) .
$$

Note $g\left(m \oplus m^{\prime}\right)=g(m)+g\left(m^{\prime}\right)$. Moreover if $m=(M, b, q)$ has a Lagrangian $L$, then by Proposition $5.2(2), T(m) \subset L$, and so $L / T(m)$ is a Lagrangian for $m_{T(m)}$. Therefore $g(m)=0$. Therefore $g$ induces a well defined homomorphism,

$$
G_{n}: \mathcal{L}^{\varepsilon}\left(R, 2^{n}\right) \rightarrow \mathcal{L}^{\mathrm{ev}}\left(R, 2^{n}\right), \quad G_{n}([m])=\left[m_{T(m)}\right] .
$$

Since $m$ and $m_{T(m)}$ share the same Witt class in $\mathcal{L}^{\varepsilon}\left(R, 2^{n}\right), \iota_{n} G_{n}=\mathrm{Id}$. When $[m] \in \mathcal{L}^{\mathrm{ev}}\left(R, 2^{n}\right)$, note $T(m)=0, m=m_{T(m)}$, and so $G_{n} \iota_{n}=\mathrm{Id}$. Therefore $G_{n}$ is the inverse of $\iota_{n}$.

(2) Suppose $n \geq 2$. We construct an inverse $F_{n}: \mathcal{L}^{\mathrm{ev}}\left(R, 2^{n}\right) \rightarrow \mathcal{L}\left(R, 2^{n-1}\right)$ to $j_{n-1}$. Let $m=(M, b, q) \in \Lambda^{\mathrm{ev}}\left(R, 2^{n}\right)$. Then

$$
S(m)=\overline{2^{n-1} M}
$$

is a subLagrangian. Its subLagrangian construction $m_{S(m)}$ has exponent $2^{n-1}$ because $2^{n-1}\left(S(m)^{\perp}\right) \subset 2^{n-1} M \subset S(m)$. We therefore define a homomorphism

$$
f: \Lambda^{\mathrm{ev}}\left(R, 2^{n}\right) \rightarrow \mathcal{L}\left(R, 2^{n-1}\right) ; \quad f(m)=\left[m_{S(m)}\right] .
$$

Claim A Let $S$ be any subLagrangian of $m \in \Lambda^{\mathrm{ev}}\left(R, 2^{n}\right)$ such that

$$
2^{n-1} S^{\perp} \subset S \subset S(m) .
$$

Then $f(m)=\left[m_{S}\right]$. (The first inclusion ensures that $\left.m_{S} \in \Lambda\left(R, 2^{n-1}\right)\right)$. 
Proof of Claim A A Lagrangian for

$$
\left(S^{\perp} / S, b_{S}, q_{S}\right) \oplus\left(S(m)^{\perp} / S(m),-b_{S(m)},-q_{S(m)}\right)
$$

is

$$
\Delta=\left\{[x] \oplus[x]: x \in S(m)^{\perp}\right\} .
$$

The second inclusion of (9) ensures that $\Delta \subset S^{\perp} / S \oplus S(m)^{\perp} / S(m)$. This proves Claim A.

Claim B If $m \in \Lambda^{\mathrm{ev}}\left(R, 2^{n}\right)$ and $m$ has a Lagrangian, then $f(m)=0$.

Proof of Claim B Suppose $m=(M, b, q)$ is a quadratic linking form, and $2^{n} M=0,2^{n} q=0$ and $L$ is a Lagrangian for $m$. We must prove $f(m)=0$.

Let $S=L \cap S(m)$, where $S(m)=\overline{2^{n-1} M}$. Obviously $S \subset S(m)$, and $L / S$ is a Lagrangian for $m_{S}$. Therefore, by Claim A, we can show $f(m)=\left[m_{S}\right]=0$ by showing $2^{n-1} S^{\perp} \subset S$, which we now do.

By Proposition A.6. $\overline{2^{n-1} M}=\left(2^{n-1} M\right)^{\perp \perp}=\left({ }_{\left.2^{n-1} M\right)^{\perp}}\right.$. So,

$$
\begin{aligned}
S^{\perp}=\left(L \cap \overline{2^{n-1} M}\right)^{\perp}=\left(L^{\perp} \cap \overline{2^{n-1} M}\right)^{\perp}= & \left(L^{\perp} \cap\left({ }_{2^{n-1}} M\right)^{\perp}\right)^{\perp} \\
& =\left(L+{ }_{2^{n-1}} M\right)^{\perp \perp}=\overline{L+{ }_{2^{n-1} M} .}
\end{aligned}
$$

Therefore, $\quad 2^{n-1} S^{\perp}=2^{n-1}\left(\overline{L+{ }_{2} n-1} M\right) \subset \overline{2^{n-1} L} \subset L \cap \overline{2^{n-1} M}=S$.

This proves $2^{n-1} S^{\perp} \subset S$, and therefore proves Claim B.

By Claim B, $f$ induces a homomorphism:

$$
F_{n}: \mathcal{L}^{\mathrm{ev}}\left(R, 2^{n}\right) \rightarrow \mathcal{L}\left(R, 2^{n-1}\right) ; \quad F_{n}([m])=\left[m_{S(m)}\right] .
$$

Since $m$ and $m_{S(m)}$ share the same Witt class in $\mathcal{L}^{\mathrm{ev}}\left(R, 2^{n}\right)$, we see $j_{n-1} F_{n}=$ Id. If $[m] \in \mathcal{L}\left(R, 2^{n-1}\right)$, then $S(m)=0$ and $m=m_{S(m)}$. So $F_{n} j_{n-1}=\mathrm{Id}$. This shows $F_{n}$ is inverse to $j_{n-1}$.

Proof of Theorem 5.1 By the definition and Lemmas 5.7 5.6(1), $\mathcal{L}(R,\langle 2\rangle)$ is the direct limit of the maps $\iota_{n+1} \circ j_{n}: \mathcal{L}\left(R, 2^{n}\right) \rightarrow \mathcal{L}\left(R, 2^{n+1}\right)$, and these maps are isomorphisms for $n \geq 2$. So $i_{n}: \mathcal{L}\left(R, 2^{n}\right) \rightarrow \mathcal{L}(R,\langle 2\rangle)$ is an isomorphism if $n \geq 2$. Define

$$
Q: \mathcal{L}(R,\langle 2\rangle) \rightarrow \mathbb{Z}_{2} \text { by: } Q \circ i_{2}=Q_{0}^{(2)}: \mathcal{L}(R, 4) \rightarrow \mathbb{Z}_{2} .
$$

From Lemmas 5.7 and 5.6, the following sequence is exact

$$
0 \rightarrow \mathcal{L}(R, 2) \stackrel{i_{1}}{\rightarrow} \mathcal{L}(R,\langle 2\rangle) \stackrel{Q}{\longrightarrow} \mathbb{Z}_{2} \rightarrow 0 .
$$


Proof of Theorem 1.5 Let $R=\mathbb{Z}[t]$ or $\mathbb{Z}\left[t^{-}\right]$. From Theorem 5.1 we get a commutative diagram with exact rows, whose vertical maps are induced by the augmentation $\varepsilon_{0}$ :

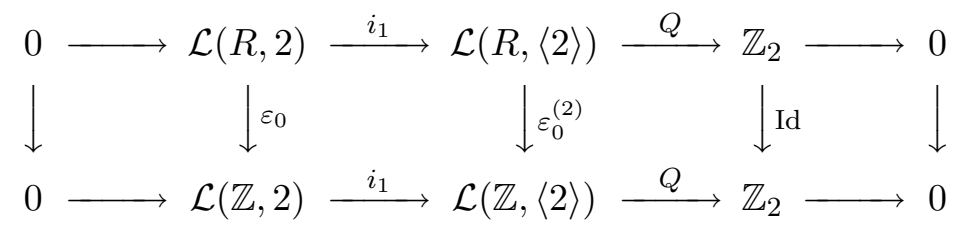

Since $\widetilde{\mathcal{L}}(R, 2)=\operatorname{ker} \varepsilon_{0}$ and $\widetilde{\mathcal{L}}(R,\langle 2\rangle)=\operatorname{ker} \varepsilon_{0}^{(2)}$, a simple diagram chase yields $i_{1}: \widetilde{\mathcal{L}}(R, 2) \cong \widetilde{\mathcal{L}}(R,\langle 2\rangle)$.

\section{Proof of Theorem 1.7}

In this section we prove Theorem 1.7 by computing $\widetilde{\mathcal{L}}\left(\mathbb{Z}\left[t^{ \pm}\right], 2\right)$, the reduced Witt group of quadratic linking forms over $\mathbb{Z}\left[t^{ \pm}\right]$of exponent 2 .

We begin with a piece of notation: For $p(t) \in \mathbb{Z}_{2}[t]$, define $p_{\text {od }}(t), p_{\text {ev }}(t) \in \mathbb{Z}_{2}[t]$ by the equation

$$
p=p_{\text {ev }}^{2}+t p_{\text {od }}^{2}=p_{\text {ev }}\left(t^{2}\right)+t p_{\text {od }}\left(t^{2}\right) \in \mathbb{Z}_{2}[t] .
$$

Definition 6.1 Let $[m] \in \widetilde{\mathcal{L}}(\mathbb{Z}[t], 2)$. Set $v_{i}=v_{i}^{(1)}([m])$. Define

$$
\begin{gathered}
B=\left(B_{1}, B_{2}\right): \widetilde{\mathcal{L}}\left(\mathbb{Z}\left[t^{ \pm}\right], 2\right) \rightarrow t \mathbb{Z}_{2}[t] \times t \mathbb{Z}_{2}[t] \\
B_{1}([m])=q\left(v_{0}\right)+t q\left(v_{1}\right) ; \\
B_{2}([m])=\left(t\left(q\left(v_{1}\right)_{\text {od }}\right)\right)^{2}+t\left(q\left(v_{0}\right)_{\text {od }}\right)^{2} .
\end{gathered}
$$

Note $B$ is a homomorphism. By (17), $\widetilde{\mathcal{L}}^{\varepsilon}(\mathbb{Z}[t], 2)=\operatorname{ker}(B)$. (Indeed, if $B_{2}([m])=$ 0 , then $q\left(v_{i}\right)=\left(q\left(v_{i}\right)_{\mathrm{ev}}\right)^{2}$ for $i=0,1$. If, in addition, $B_{1}([m])=0$, then $q\left(v_{0}\right)$ and $q\left(v_{1}\right)$ are zero.) By Lemma[5.6], $B$ takes values in $\widehat{H}_{1}\left(\mathbb{Z}_{2} ; R\right) \times \widehat{H}_{1}\left(\mathbb{Z}_{2} ; R\right) t=$ $\mathbb{Z}_{2}[t] \times t \mathbb{Z}_{2}[t]$. But since $\varepsilon_{0} \circ Q^{(1)}=Q^{(1)} \circ \varepsilon_{0 *}$, and $\varepsilon_{0 *}([m])=0, B$ takes values in $t \mathbb{Z}_{2}[t] \times t \mathbb{Z}_{2}[t]$.

The following example shows that $\widetilde{\mathcal{L}}(\mathbb{Z}[t], 2) \stackrel{B}{\rightarrow} t \mathbb{Z}_{2}[t] \times t \mathbb{Z}_{2}[t]$ is an epimorphism.

Example 6.2 Let $p, g \in \mathbb{Z}[t]$. For the quadratic linking form

$$
\mathcal{N}_{p, g}=\left(\mathbb{Z}_{2}[t]^{2},\left(\begin{array}{cc}
p / 2 & 1 / 2 \\
1 / 2 & 0
\end{array}\right),\left(\begin{array}{c}
p / 2 \\
g
\end{array}\right)\right)
$$


we have $v_{0}=\left(0, p_{\text {ev }}\right)$ and $v_{1}=\left(0, p_{\text {od }}\right)$ by equation (44). Hence,

$$
\begin{aligned}
q\left(v_{0}\right)=p_{\mathrm{ev}}^{2} g, q\left(v_{1}\right) & =p_{\mathrm{od}}^{2} g . \\
B\left(\left[\mathcal{N}_{p, g}\right]\right) & =\left(p g,\left(g_{\mathrm{od}}\right)^{2} t p\right) . \quad \text { In particular } \\
B\left(\left[\mathcal{N}_{t p, 1}\right]\right) & =(t p, 0) \\
B\left(\left[\mathcal{N}_{1, t p}\right]-\left[\mathcal{N}_{t, p}\right]\right) & =(0, t p) .
\end{aligned}
$$

\section{Proof of Theorem 1.7}

(1) The above discussion, together with Lemma 5.7(1) and Theorem 4.8(1) (where $\mathcal{P}$ is defined), show that the following sequence is exact:

$$
0 \rightarrow t \mathbb{Z}_{2}[t] /\left(V_{2}-1\right) t \mathbb{Z}_{2}[t] \stackrel{\iota_{1} \circ \mathcal{P}}{\longrightarrow} \widetilde{\mathcal{L}}(\mathbb{Z}[t], 2) \stackrel{B}{\longrightarrow} t \mathbb{Z}_{2}[t] \times t \mathbb{Z}_{2}[t] \rightarrow 0 .
$$

In particular, $\widetilde{\mathcal{L}}(\mathbb{Z}[t], 2)$ is generated by those elements $\left[\mathcal{N}_{p, g}\right]$, for which either $p$ or $q$ is divisible by $t$ (cf Lemma 4.3(3)). By Lemma 4.3 parts (2), (3), and (4), the map

$$
j_{1}: t \mathbb{Z}_{4}[t] /\left(2 V_{2}-2\right) t \mathbb{Z}_{4}[t] \rightarrow \widetilde{\mathcal{L}}(\mathbb{Z}[t], 2) ; \quad j_{1}[t p]=\left[\mathcal{N}_{t p, 1}\right]
$$

is a well-defined homomorphism of abelian groups. It satisfies $j_{1}[2 t p]=\iota_{1} \mathcal{P}[t p]$. It is clearly a $\mathcal{V}$-map. By Example 6.2.

$$
B_{1} j_{1}[t p]=[t p] ; \quad B_{2} j_{1}[t p]=0 .
$$

Therefore the following sequence is exact.

$$
0 \rightarrow t \mathbb{Z}_{4}[t] /\left(2 V_{2}-2\right) t \mathbb{Z}_{4}[t] \stackrel{j_{1}}{\longrightarrow} \widetilde{\mathcal{L}}(\mathbb{Z}[t], 2) \stackrel{B_{2}}{\longrightarrow} t \mathbb{Z}_{2}[t] \rightarrow 0
$$

We next claim that the map $B_{2}: \widetilde{\mathcal{L}}(\mathbb{Z}[t], 2) \rightarrow\left[t \mathbb{Z}_{2}[t]\right]$ is a $\mathcal{V}$-map, where $\left[t \mathbb{Z}_{2}[t]\right]$ is the $\mathcal{V}$-module defined by (2). In fact,

$$
\begin{aligned}
V_{2 n+1} B_{2}\left(\left[\mathcal{N}_{p, g}\right]\right) & =t p\left(t^{2 n+1}\right)\left(t^{n} g_{\text {od }}\left(t^{2 n+1}\right)\right)^{2}= \\
B_{2}\left(\left[\mathcal{N}_{p\left(t^{2 n+1}\right), g\left(t^{2 n+1}\right)}\right]\right) & =B_{2} V_{2 n+1}\left(\left[\mathcal{N}_{p, g}\right]\right) ; \\
V_{2} B_{2}\left(\left[\mathcal{N}_{p, g}\right]\right)=0 & =B_{2}\left(\left[\mathcal{N}_{p\left(t^{2}\right), g\left(t^{2}\right)}\right]\right)=B_{2} V_{2}\left(\left[\mathcal{N}_{p, g}\right]\right) .
\end{aligned}
$$

proving the claim. Therefore (15) becomes an exact sequence of $\mathcal{V}$-modules. A right splitting for it is the map

$$
j_{2}:\left[t \mathbb{Z}_{2}[t]\right] \rightarrow \widetilde{\mathcal{L}}(\mathbb{Z}[t], 2) ; \quad j_{2}[t p]=\left[\mathcal{N}_{1, t p}\right]-\left[\mathcal{N}_{t, p}\right] .
$$

Note $j_{2}[t p+t 2 q]=\left[\mathcal{N}_{1, t p}\right]-\left[\mathcal{N}_{t, p}\right]=j_{2}[t p]$, so $j_{2}$ is well defined. Similarly, $j_{2}$ is a homomorphism because

$$
j_{2}[t p]+j_{2}[t q]-j_{2}[t p+t q]=\left[\mathcal{P}_{t p, t q}\right]-\left[\mathcal{P}_{t^{2} p, q}\right]=0,
$$


by Lemmas 4.3(8) and 4.3(6). It is a splitting by equation (14). It is a $\mathcal{V}$-map by Lemma 4.3(7) and the following calculation:

$$
\begin{aligned}
& V_{2}\left(j_{2}(t p)\right)=\left[\mathcal{N}_{1, t^{2} p^{2}}\right]-\left[\mathcal{N}_{t^{2}, p^{2}}\right]=0 \text { by Lemma 4.3(5). } \\
& j_{2} V_{2}(t p)=0 \text {, for all } t p \in\left[t \mathbb{Z}_{2}[t]\right] \text { by (2). } \\
& V_{2 n+1}\left(j_{2}(t p)\right)=\left[\mathcal{N}_{1, t^{2 n+1} p\left(t^{2 n+1}\right)}\right]-\left[\mathcal{N}_{t^{2 n+1}, p\left(t^{2 n+1}\right)}\right] \\
& =\left[\mathcal{N}_{1, t^{2 n+1} p\left(t^{2 n+1}\right)}\right]-\left[\mathcal{N}_{t, t^{2 n} p\left(t^{2 n+1}\right)}\right] \text { by Lemma 4.3. }(5) \text {. } \\
& =j_{2} V_{2 n+1}(t p) \text { by (2). }
\end{aligned}
$$

Therefore, we get an isomorphism of $\mathcal{V}$-modules, proving Theorem [1.7)(1),

$$
j_{1}+j_{2}: \frac{t \mathbb{Z}_{4}[t]}{\left(2 V_{2}-2\right) t \mathbb{Z}_{4}[t]} \oplus\left[t \mathbb{Z}_{2}[t]\right] \rightarrow \widetilde{\mathcal{L}}(\mathbb{Z}[t],\langle 2\rangle) .
$$

(2) If $[m] \in \widetilde{\mathcal{L}}\left(\mathbb{Z}\left[t^{-}\right], 2\right)$, then $q\left(v_{0}^{(1)}(m)\right) \in t^{2} \mathbb{Z}_{2}\left[t^{2}\right]$, since $\varepsilon_{0} q\left(v_{0}^{(1)}(m)\right)=$ 0 and $q\left(v_{0}^{(1)}(m)\right) \in \widehat{H}_{1}\left(\mathbb{Z}_{2} ; \mathbb{Z}\left[t^{-}\right]\right)=\mathbb{Z}_{2}\left[t^{2}\right]$, by Lemma [5.6] (3). The map $Q_{0}^{(1)}: \widetilde{\mathcal{L}}\left(\mathbb{Z}\left[t^{-}\right], 2\right) \rightarrow t^{2} \mathbb{Z}_{2}\left[t^{2}\right]$ is a homomorphism by Lemma 4.3 (2). The map $Q_{0}^{(1)}$ is injective by Theorem 4.8(2) and Lemma 5.7(1). In fact $Q_{0}^{(1)}$ is an isomorphism because $Q_{0}^{(1)}\left(\left[\mathcal{N}_{t^{2} p\left(t^{2}\right), 1}\right]\right)=t^{2} p\left(t^{2}\right)$, by equation (12). The inverse isomorphism to $Q_{0}^{(1)}$ is the $\mathcal{V}_{\text {od }}-$ map:

$$
j: t^{2} \mathbb{Z}_{2}\left[t^{2}\right] \rightarrow \widetilde{\mathcal{L}}\left(\mathbb{Z}\left[t^{-}\right], 2\right) ; \quad j\left(t^{2} p\left(t^{2}\right)\right)=\left[\mathcal{N}_{t^{2} p\left(t^{2}\right), 1}\right] .
$$

The reader may note that $\mathrm{UNil}_{1}(\mathbb{Z} ; \mathbb{Z}, \mathbb{Z})$ has not been discussed here. In fact it is already known to be zero. See [7, or [10, or [1]. But for the reader's convenience we include a short proof.

Proposition 6.3 $\mathrm{UNil}_{1}(\mathbb{Z} ; \mathbb{Z}, \mathbb{Z})=0$.

Proof By Theorem 1.1 and Proposition $1.4 . \mathrm{UNil}_{1}(\mathbb{Z} ; \mathbb{Z}, \mathbb{Z})=N L_{1}(\mathbb{Z})=$ $N L_{1}(\mathbb{Z},\langle 2\rangle)$, which can be identified with a subgroup of

$$
\mathcal{L}^{-}(\mathbb{Z}[t],\langle 2\rangle)=Q W\left(\mathbb{Z}[t],(\mathbb{Q}[t] / \mathbb{Z}[t])^{-}, \mathbf{M}\langle 2\rangle\right),
$$

the Grothendieck group of skew quadratic linking forms over $\mathbb{Z}[t]$-modules $M$ with $2^{n} M=0$ for some $n$.

We will show $\mathcal{L}^{-}(\mathbb{Z}[t],\langle 2\rangle)=0$. Let $R=\mathbb{Z}[t]$. For any $\left(R,(\mathbb{Q} R / R)^{-}, \mathbf{M}\langle 2\rangle\right)-$ form, say $m=(M, \lambda, \mu)$, we see $\mu: M \rightarrow \frac{(\mathbb{Q} R / R)}{(1-*)(\mathbb{Q} R / R)}=\frac{(\mathbb{Q} R / R)}{2(\mathbb{Q} R / R)}=0$. So $\mu(x)=0$, and $\lambda(x, x)=0$ for all $x \in M$. If $2^{n} M=0, n \geq 2$, this implies that $S=\overline{2^{n-1} M}$ is a subLagrangian for $m$, and $m_{S}$ has exponent $2^{n-1}$. And if $2 M=0$, it implies that for any $x \neq 0$ in $M, S=\overline{\langle x\rangle}$ is a subLagrangian for $m$, with $\operatorname{rank}_{R / 2 R}\left(m_{S}\right) \leq \operatorname{rank}_{R / 2 R}(m)-1$. Of course this means $[m]=0$, as desired. 


\section{A Appendix: On length and colength and the sub- Lagrangian construction}

Here we prove several properties used in the text about the colength of an $R$ submodule. Length and colength were defined in Section 2, We also prove that the subLagrangian construction produces a nonsingular form in the same Witt class.

Proposition A.1 Let $R$ be a ring with involution which is torsion free as an abelian group. Then for any $n,\left(R, \mathbb{Q} R / R, \mathbf{M}_{2^{n}}\right)$ satisfies the conditions of Definition 2.1. where $\mathbf{M}_{2^{n}}$ is the class of length one $R$-modules $M$ with $2^{n} M=0$.

Proof 2.1(2) is obvious.

The first part of 2.1(3) is a standard fact from homological algebra. The proof of the second part of $2.1(3)$ amounts to showing that $\operatorname{Ext}_{R}^{1}(M, \mathbb{Q} R / R)=0$ for any $M \in \mathbf{M}_{2^{n}}$. But $\operatorname{Ext}_{R}^{i}(M, \mathbb{Q} R)=0$ for all $i \geq 0$, because $2^{n} M=$ 0 and $\times 2^{n}: \mathbb{Q} R \rightarrow \mathbb{Q} R$ is an isomorphism. Therefore $\operatorname{Ext}_{R}^{1}(M, \mathbb{Q} R / R) \cong$ $\operatorname{Ext}_{R}^{2}(M, R)=0$, because $M$ has length one.

Now we prove 2.1(1). Let $M \in \mathbf{M}_{2^{n}}$. Clearly $2^{n} M^{\wedge}=0$. There is an exact sequence $0 \rightarrow F_{1} \rightarrow F_{0} \rightarrow M \rightarrow 0$, where $F_{0}, F_{1}$ are finitely generated and free. Since $M^{*}:=\operatorname{Hom}_{R}(M, R)=0$, we get a resulting exact sequence $0 \rightarrow F_{0}^{*} \rightarrow$ $F_{1}^{*} \rightarrow \operatorname{Ext}_{R}^{1}(M, R)=M^{\wedge} \rightarrow 0$. This shows $M \in \mathbf{M}_{2^{n}}$. Let $d: F \rightarrow F^{* *}$ denote the double duality isomorphism for any finitely generated free $R$-module. By dualizing the above short exact sequence a second time, we get a commutative diagram with exact rows:

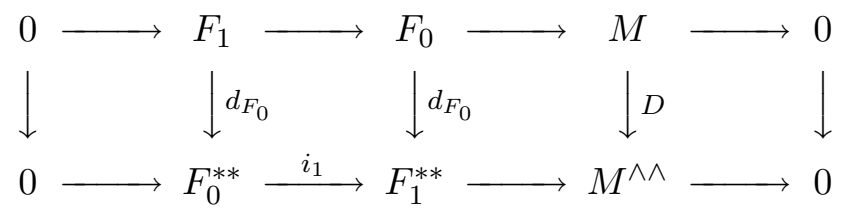

where $D$ denotes the double duality map. Since $d_{F_{i}}$ is an isomorphism for $i=0,1$, we conclude that $D$ is too.

Lemma A.2 Let $(R, A, \mathbf{M})$ be any triple satisfying the conditions of Definition 2.1. Let $S$ be a subLagrangian for some $(R, A, \mathbf{M})$-form $m=(M, \lambda, \mu)$. Then $m_{S}$ (as defined in Section [2) is a $(R, A, \mathbf{M})$-form, and $[m]=\left[m_{S}\right]$ in $Q W(R, A, \mathbf{M})$. 
Proof Since $M, M / S \in \mathbf{M}$ and $0 \rightarrow S \stackrel{i}{\rightarrow} M \rightarrow M / S \rightarrow 0$ is exact, we see $S \in \mathbf{M}$ and $M^{\wedge} \rightarrow S^{\wedge}$ is surjective. Consider the commutative diagram, where $j$ and $k$ denote inclusions:

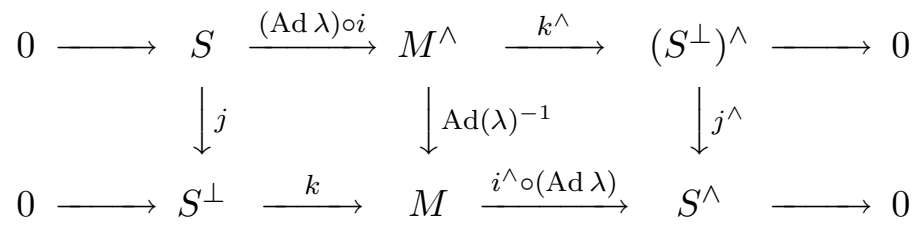

The bottom row is exact, and thus, by 2.11(3), so is its dual, the top row. The exact sequence $0 \rightarrow S^{\perp} / S \rightarrow M / S \rightarrow M / S^{\perp} \rightarrow 0$ and the isomorphism $M / S^{\perp} \cong S^{\wedge} \in \mathbf{M}$ show $S^{\perp} / S \in \mathbf{M}$ and hence $\operatorname{ker}\left(j^{\wedge}\right) \cong\left(S^{\perp} / S\right)^{\wedge}$. The Snake Lemma isomorphism $S^{\perp} / S=\operatorname{cok}(j) \cong \operatorname{ker}\left(j^{\wedge}\right)=\left(S^{\perp} / S\right)^{\wedge}$ can be identified with $\operatorname{Ad} \lambda_{S}$. So $m_{S}$ is nonsingular and is therefore a $(R, A, \mathbf{M})$-form. To see $[m]=\left[m_{S}\right]$, note $m_{S} \oplus(-m)$ has a Lagrangian; it is $\left\{([x], x) \in\left(S^{\perp} / S\right) \times M\right.$ : $\left.x \in S^{\perp}\right\}$.

Throughout the rest of this appendix, $R$ denotes $\mathbb{Z}[t]$.

Proposition A.3 Let $M$ be a finitely generated $R$-module of exponent $2^{n}$ for some $n$. Then $M$ has length one if and only if for each $x \neq 0$ in $M$, the annihilator ideal satisfies $\operatorname{Ann}_{R}(x) \subseteq 2 R$.

Proof $\Rightarrow$ : We will prove the contrapositive. Supposing that $x \neq 0$ in $M$ and $\operatorname{Ann}_{R}(x) \nsubseteq 2 R$, we will show that $\operatorname{Length}_{R}(M) \geq 2$.

Replacing $x$, if necessary, by $2^{k} x$, for some $k$, we see that we may as well assume that $2 x=0$. Since $\mathbb{Z}_{2}[t]$ is a principal ideal domain, $\operatorname{Ann}_{R}(x)=\langle 2, p\rangle$ for some $p \in R \backslash 2 R$. Let $f: \operatorname{Ann}_{R}(x) \rightarrow \mathbb{Q}[t] / \mathbb{Z}[t]$ be the $R$-map such that $f(2)=0$, and $f(p)=\frac{1}{2}$. $f$ does not extend to an $R$-map, $R \rightarrow \mathbb{Q}[t] / \mathbb{Z}[t]$. Therefore the exact sequence, $0 \rightarrow \operatorname{Ann}_{R}(x) \rightarrow R \rightarrow R x \rightarrow 0$ shows that $0 \neq \operatorname{Ext}_{R}^{1}(R x, \mathbb{Q}[t] / \mathbb{Z}[t]) \cong \operatorname{Ext}_{R}^{2}(R x, \mathbb{Z}[t])$. Since $\operatorname{Ext}_{R}^{3}(-,-)=0$, the exact sequence $0 \rightarrow R x \rightarrow M \rightarrow M / R x \rightarrow 0$ shows that $\operatorname{Ext}_{R}^{2}(M, \mathbb{Z}[t]) \neq 0$. This implies that $\operatorname{Length}_{R}(M) \geq 2$ as claimed.

$\Leftarrow$ : Now assume for each $x \neq 0$ in $M$, that $\operatorname{Ann}_{R}(x) \subseteq 2 R$. We show that Length $_{R}(M)=1$. The proof is by induction on $n$. If $n=1$ the condition implies $M$ is a free $R / 2 R$-module (because it is $R / 2 R$-torsion free). This implies Length ${ }_{R}(M)=1$. Assume the result is known for modules of exponent $2^{n-1}$. Consider the exact sequence

$$
0 \rightarrow{ }_{2} M \rightarrow M \rightarrow M /{ }_{2} M \rightarrow 0,
$$


where ${ }_{2} M=\{x \in M: 2 x=0\}$. For any $x \in M$ with $[x] \neq 0$ in $M /{ }_{2} M$ we note $\operatorname{Ann}_{R}([x]) \subseteq \operatorname{Ann}_{R}(2 x) \subseteq 2 R$. This implies, by the inductive hypothesis, that $\operatorname{Length}_{R}\left(M /{ }_{2} M\right)=1$, and also Length ${ }_{R}\left({ }_{2} M\right)=1$. It follows that Length $_{R}(M)=1$.

Corollary A.4 Let $M$ be a finitely generated $R$ module of exponent $2^{n}$ with length one. Any submodule of $M$ has length one. Also $M^{\wedge}$ has length one.

Proof The first claim is immediate from Proposition A.3. To see that $M^{\wedge}$ has length one, one applies Proposition A.3, noting that the annihilator ideal of any element of $2^{n}\left(\mathbb{Q}[t] / \mathbb{Z}[t]\right.$ ) (and therefore any element of $M^{\wedge}$ ) is principal, generated by $2^{k}$ for some $k$.

Recall the definition of the closure of a submodule $N$ of a length one module $M$ :

$$
\bar{N}=\{x \in M: p x \in N, \text { for some } p \in R \backslash 2 R\}
$$

Clearly $\overline{\bar{N}}=\bar{N}$. By Proposition A.3. $\operatorname{Length}_{R}(M / N)=1$ if and only if $N=\bar{N}$.

Corollary A.5 Let $N$ be a submodule of a length one $R$-module $M$. Then $N$ has colength one in $M$ if and only if $N=\bar{N}$. In particular, $\bar{N}$ has colength one.

Proposition A.6 Let $M$ be a length one $R$-module of exponent $2^{n}$. Let $b: M \times M \rightarrow \mathbb{Q}[t] / \mathbb{Z}[t]$ be a nonsingular linking form (relative to some involution on $R$ ). Let $N$ be a submodule of $M$. Then $N$ has colength one in $M$ if and only if $N=X^{\perp}$ for some $X$ in $M$. Also $\bar{N}=N^{\perp \perp}$ for any $N$.

Proof Suppose $X$ is a submodule of $M$ for which $N=X^{\perp}$. Then

$$
0 \rightarrow N \rightarrow M \stackrel{i_{X}^{\circ}(\operatorname{Ad} b)}{\longrightarrow} X^{\wedge}
$$

is exact, where $i_{X}: X \rightarrow M$ denotes the inclusion. But $\operatorname{im}\left(i_{X}^{\wedge} \circ(\operatorname{Ad} b)\right)$ has length one by Corollary A.5. Therefore $N$ has colength one in $M$.

Conversely, suppose $N$ has colength one in $M$. By Proposition A.1, this implies $M^{\wedge} \stackrel{i_{N}^{\wedge}}{\longrightarrow} N^{\wedge} \rightarrow 0$ is exact. Set $X=\operatorname{ker}\left(i_{N}^{\wedge} \circ(\operatorname{Ad} b)\right)$. This gives us an exact sequence and its dual:

$$
\begin{aligned}
& 0 \rightarrow X \stackrel{i_{X}}{\longrightarrow} M \stackrel{i_{N}^{\wedge} \circ(\operatorname{Ad} b)}{\longrightarrow} N^{\wedge} \rightarrow 0 . \\
& 0 \rightarrow N \stackrel{i_{N}}{\longrightarrow} M \stackrel{i_{X}^{\circ} \circ(\operatorname{Ad} b)}{\longrightarrow} X^{\wedge} \rightarrow 0 .
\end{aligned}
$$


The second sequence says that $N=X^{\perp}$.

Finally we show $\bar{N}=N^{\perp \perp}$. By the definition of $\bar{N}$, it is clear that $\bar{N}^{\perp}=N^{\perp}$. But we have just seen that $\bar{N}=X^{\perp}$, for some $X \subset M$. Moreover, $X^{\perp \perp \perp}=X^{\perp}$ for all $X$. So $N^{\perp \perp}=\bar{N}^{\perp \perp}=X^{\perp \perp \perp}=X^{\perp}=\bar{N}$.

Acknowledgement This work is partially supported by the National Science Foundation.

\section{References}

[1] C Arf, Untersuchungen Über quadratische Formen in Körpern der Charakteristik 2. I, J. Reine Angew. Math. 183 (1941) 148-167 MathReview

[2] H Bass, Algebraic K-theory, Benjamin (1968) MathReview

[3] M Banagl, A Ranicki, Generalized Arf invariants in algebraic L-theory, arXiv:math.AT/0304362

[4] W Browder, Surgery on Simply Connected Manifolds, Ergebnisse series 65, Springer-Verlag (1972) MathReview

[5] SE Cappell, Splitting obstructions for hermitian forms and manifolds with $\mathbb{Z}_{2} \subset \pi_{1}$, Bull. Amer. Math. Soc. 79 (1973) 909-913 MathReview

[6] S E Cappell, On connected sums of manifolds, Topology 13 (1974) 395-400 MathReview

[7] S E Cappell, Unitary nilpotent groups and hermitian K-theory. I, Bull. Amer. Math. Soc. 80 (1974) 1117-1122 MathReview

[8] S E Cappell, Manifolds with fundamental group a generalized free product. I, Bull. Amer. Math. Soc. 80 (1974) 1193-1198 MathReview

[9] S E Cappell, A splitting theorem for manifolds, Invent. Math. 33 (1976) 69-170 MathReview

[10] F X Connolly, T Koźniewski, Nil groups in $K$-theory and surgery theory, Forum Math. 7 (1995) 45-76 MathReview

[11] F Connolly, A A Ranicki, On the calculation of UNil, to appear in Advances in Math. arXiv:math.AT/0304016

[12] F T Farrell, The exponent of UNil, Topology 18 (1979) 305-312 MathReview

[13] F T Farrell, L E Jones, Isomorphism conjectures in algebraic $K$-theory, J. Amer. Math. Soc. 6 (1993) 249-297 MathReview

[14] I Hambleton, L R Taylor, A guide to the calculation of the surgery obstruction groups for finite groups, from: "Surveys on surgery theory, Vol. 1", Ann. of Math. Stud. 145, Princeton Univ. Press (2000) 225-274 MathReview 
[15] M A Kervaire, J H Milnor, Groups of homotopy spheres. I, Ann. of Math. 77 (1963) 504-537 MathReview

[16] A A Ranicki, Algebraic L-theory. I. Foundations, Proc. London Math. Soc. 27 (1973) 101-125 MathReview

[17] A A Ranicki, Algebraic L-theory II. Laurent extensions, Proc. London Math. Soc. 27 (1973) 126-158 MathReview

[18] A A Ranicki, Algebraic L-theory. III. Twisted Laurent extensions, from: "Algebraic K-theory, III: Hermitian K-theory and geometric application" (Proc. Conf. Seattle Res. Center, Battelle Memorial Inst. 1972) Lecture Notes in Math. Vol. 343, Springer (1973) 412-463 MathReview

[19] A A Ranicki, Algebraic L-theory. IV. Polynomial extension rings, Comment. Math. Helv. 49 (1974) 137-167 MathReview

[20] A A Ranicki, The algebraic theory of surgery. I. Foundations, Proc. London Math. Soc. 40 (1980) 87-287 MathReview

[21] A A Ranicki, Exact sequences in the algebraic theory of surgery, Mathematical Notes 26, Princeton Univ. Press (1981) MathReview

[22] A A Ranicki, Letter to Frank Connolly, (1996) http://www.maths.ed.ac.uk/ aar/surgery/andrew.pdf

[23] A A Ranicki, High dimensional knot theory. Algebraic surgery in codimesnsion 2, with an appendix by Elmar Winkelnkemper, Springer Monographs series, Springer-Verlag (1998) MathReview

[24] W Scharlau, Quadratic and Hermitian Forms, Grundlehren series 270, Springer-Verlag (1985) MathReview

[25] J L Shaneson, Wall's surgery obstruction groups for $G \times \mathbb{Z}$, Ann. of Math. 90 (1969) 296-334 MathReview

[26] J Stallings, Whitehead torsion of free products, Ann. of Math. 82 (1965) 354363 MathReview

[27] F Waldhausen, Algebraic $K$-theory of generalized free products. I, II, III, IV, Ann. of Math. 108 (1978) 135-256 MathReview

[28] C T C Wall, Surgery of non-simply-connected manifolds, Ann. of Math. 84 (1966) 217-276 MathReview

[29] C T C Wall, Surgery on Compact Manifolds, 2nd edition (edited and with a foreword by A A Ranicki) Math. Surveys and Monographs 69, A.M.S. (1999) MathReview 